\title{
POLÍTICA SEXUAL Y REPRODUCCIÓN SOCIAL EN LA PAMPA DEL TAMARUGAL: ESTRUCTURA SEXO-EDAD EN EL CEMENTERIO TARAPACÁ 40 (1000 AC-600 DC)
}

\author{
SEXUAL POLITICS AND SOCIAL REPRODUCTION IN THE PAMPA \\ DEL TAMARUGAL: SEX-AGE STRUCTURE IN THE TARAPACÁ 40 \\ CEMETERY (BC 1000-600 AD)
}

\author{
Andrea González-Ramírez ${ }^{1,2,3}$, Arturo Sáez ${ }^{2,3}$, María José Herrera Soto ${ }^{4}$, Lía Leyton ${ }^{5}$, \\ Felipe Miranda ${ }^{6}$, Francisca Santana-Sagredo ${ }^{7}$ y Mauricio Uribe Rodríguez ${ }^{8}$
}

\begin{abstract}
Ofrecemos hipótesis explicativas respecto a las políticas sexuales que, eventualmente, se implementaron en la configuración de la comunidad formativa de la Pampa del Tamarugal, siendo nuestro objetivo central contribuir al estudio arqueológico de la reproducción social. Las colecciones bioantropológicas del Cementerio Tarapacá 40 fueron sometidas a un relevamiento osteológico orientado al conocimiento de su estructura sexual y etaria. Se recuperó un importante número de referencias contextuales y se elaboró un análisis exploratorio de la variación en la estructura sexo-edad de algunos cementerios de la región. Los resultados muestran un cementerio que presenta todos los rangos etarios y una distribución sexual 1:1. A escala regional, se observó una distribución dicotómica entre cementerios arcaicos y formativos, en función de la proporción de mujeres adultas y personas no-adultas. Se propone que entre la sociedad arcaica tardía y la formativa, las relaciones sociales de reproducción y la división sexual del trabajo habrían experimentado transformaciones radicales, cuyo impacto más directo pudo ser el control social de la sexualidad femenina a través de la instauración de un régimen sociopolítico basado en el parentesco.
\end{abstract}

Palabras claves: Reproducción Social, política sexual, estructura sexo-edad, periodo Formativo, feminismo materialista.

In this paper we propose explanatory hypotheses about the sexual politics that were from time to time implemented in the configuration of the Pampa del Tamarugal formative community, with the aim to contribute to the archaeological study of social reproduction. The bioanthropological collections of the Tarapacá 40 Cemetery underwent an osteological survey, designed to identify their sexual and age structure. A significant number of contextual references were recovered, and an exploratory analysis of the variation in the sex-age structure of some cemeteries in the region was carried out. The results show a cemetery with all age ranges and a 1:1 sex ratio. On a regional scale, a dichotomous distribution was observed between archaic and formative cemeteries, based on the ratio between adult women and non-adults. We propose that social relations of reproduction and sexual division of labor between the late archaic and the formative society underwent radical transformations, the most direct impact of which could be the social control of female sexuality through the establishment of a kinship-based socio-political regime.

Key words: Social reproduction, sexual politics, sex-age structure, Formative period, materialist feminism.

Biológica o natural es la capacidad de las mujeres de llevar a maduración los óvulos, de llevar a cabo un embarazo iniciado, más no lo es el número de hijos dados a luz durante los siglos. Entre el óvulo y el hijo, entre la capacidad de procrear y el hecho de procrear, se hallan las relaciones entre hombres y mujeres. Entre la capacidad de procrear y la procreación, puestas en actos, está la historia de las relaciones de reproducción, en buena parte, la historia de la reproducción como explotación (Tabet 2018 [1985]:126).

1 Escuela de Arqueología, Universidad Austral de Chile, Puerto Montt, Chile. andrea.gonzalez@uach.cl

2 Grupo de Investigación ACAIA (Arqueología de las Comunidades A-estatales Ibéricas y Andinas), Universitat Autònoma de Barcelona, Barcelona, España.

3 Observatorio TREBALL, Pitrufquén, Chile. arturosaezrex@gmail.com

4 Programa de Doctorado en Arqueología, Facultad de Filosofía y Letras, Universidad de Buenos Aires, Buenos Aires, Argentina. majo.hersot@gmail.com

5 Investigadora independiente, Santiago, Chile. lialeyton.g@gmail.com

6 Carrera de Arqueología, Universidad SEK, Santiago, Chile. feigmiga@ gmail.com

7 Escuela de Antropología, Pontificia Universidad Católica de Chile, Santiago, Chile. francisca.santana@uc.cl

8 Departamento de Antropología, Universidad de Chile, Santiago, Chile. mur@uchile.cl

Recibido: abril 2019. Aceptado: agosto 2020.

http://dx.doi.org/10.4067/S0717-73562021005001801. Publicado en línea: 30-septiembre-2021. 
El proceso de reproducción social constituye un sistema complejo de tareas, trabajos y energías, cuyo objetivo es la reproducción de la población, de las relaciones sociales que la sostienen y, especialmente, de la reproducción de la fuerza de trabajo (Benería 1981; Bourdieu 2011; Carrasco et al. 2019; Dalla Costa 1975; Molyneux 1979; Picchio 2019). Su estudio, seminalmente emprendido por enfoques marxistas (Passeron y García 1983), replicó, no obstante, el razonamiento de la economía y la sociología liberales al desplazar de la vida económica y política los trabajos procreativos y de cuidados, normalizados y desvalorizados como labores feminizadas. Según la economía feminista, este sesgo es heredero de una "ideología de la domesticidad" la que situó a las mujeres como responsables naturales del cuidado y del trabajo doméstico, instalando un nuevo orden simbólico de la maternidad en conflicto con las actividades productivas (Carrasco 1991:17-21; Carrasco et al. 2019). Se trata de un fenómeno bien estudiado por historiadoras, sociólogas y antropólogas, que reconocen el inicio de la desvalorización de estas labores en el desarrollo mercantil de la industrialización capitalista (Federici 2010, 2018). Asimismo, numerosas investigaciones históricas en sociedades preindustriales han mostrado una gran variabilidad a lo largo del tiempo, tanto en los modos de organizar y normar estos trabajos, como en las formas que prevalecen sobre otras (Boydston 1990; Tabet 2018).

Uno de los elementos centrales de la reproducción social es la organización de los trabajos procreativos que, básicamente, constituyen un proceso integrado por la gestación, parto, puerperio y lactancia (Falquet 2018; Tabet 2018). Se definen como "trabajo" pues no se trata únicamente de un proceso orgánico, sino de un gasto energético que no es indispensable para la conservación del individuo reproductor y porque genera un producto externo que, como señala Paola Tabet (2018:167), no es un residuo de otro proceso de producción, sino un producto en sí. Al ser considerados como trabajos, abandonan el orden simbólico ilustrado de lo biológico como expresión de una Naturaleza esencializada, y pueden ser comprendidos en su dimensión social e histórica.

Si bien en bioantropología y arqueología se han querido mostrar como empíricas ciertas razones que justifican la prácticamente total ausencia de esta clase de preguntas (Vila-Mitjá 2011), puede afirmarse, antes bien, que derivan de una potente y silenciosa influencia de la economía neoclásica y la economía política clásica en los razonamientos arqueológicos dominantes, especialmente en aquellos enfoques interesados por asuntos económicos (Picchio 2019; Risch 2002).

En la historia de la investigación del Norte Árido de Chile aquello puede verse manifestado claramente en los modelos de control vertical de pisos ecológicos (Murra 1987), de movilidad giratoria (Núñez y Dillehay 1979) y, en general, en los que enfatizan la organización tecnológica de la producción como mecanismo para asegurar el intercambio de bienes de prestigio y la obtención de estatus (Núñez et al. 2006). En estos modelos la teoría del intercambio se basa en el principio liberal según el cual se debe optimizar el uso de recursos escasos, mientras que "el nivel de vida, la unidad doméstica, el conflicto social y las instituciones sociales se tratan como particularidades separadas" (Picchio 2019:126). Por su parte, la influencia de la economía política clásica, especialmente en la arqueología que emplea datos provenientes del trabajo bioantropológico (GonzálezRamírez y Sáez 2011), se refleja en la idea que concibe a la demografía como factor y no como producto de las relaciones sociales (MacInnes y Pérez 2008).

En este trabajo buscamos realizar un primer ejercicio exploratorio para leer en clave de reproducción social a Tarapacá-40, un cementerio que ha sido muy relevante para el estudio de la historia social y económica de la transición arcaica-formativa en la Pampa del Tamarugal en el Norte de Chile (Adán et al. 2007; Agüero y Uribe 2015). Un periodo para el que se han documentado importantes procesos de transformación en la vida social producto de cambios en los modos de producción, cuyo ritmo e intensidad si bien aún resultan inciertos, pueden ser esbozados gracias a numerosas investigaciones que han aportado evidencias desde hace más de 50 años.

Ya que los cementerios son espacios de representación social, resultan particularmente adecuados para explorar empíricamente los filtros y categorías que las organizaciones sociales sancionan para su estructuración (Gowland y Knüsel 2006; Lull y Picazo 1989; McGuire 2018; Parker Pearson 1999). De ahí que la composición sexual y etaria sea un parámetro relevante para ponderar tanto los criterios directos de selección de ingreso al espacio comunitario de la muerte, como indirectamente a las relaciones sociales y sexuales naturalizadas por la cultura.

En este estudio empleamos a la estructura sexoedad de este cementerio para formular las primeras hipótesis relativas a la política sexual implementada 
en este periodo respecto a los trabajos de procreación, como base para conocer los mecanismos de la reproducción social que debieron verse intervenidos en estas transformaciones socio-económicas (Millet 2010). Para este propósito, seleccionamos como caso de estudio al cementerio Tarapacá-40, ya que ha sido una de las colecciones arqueológicas más importantes para el estudio bioantropológico del periodo Formativo del Norte de Chile. No obstante, se trata de un cementerio solo parcialmente publicado y cuya colección arqueológica y bioantropológica se encuentra repartida en varias instituciones, provocando un mosaico desigual de información y conservación que debimos sortear a través de la implementación de una arqueología de colecciones.

En este artículo presentamos los resultados obtenidos en tres objetivos básicos:

(a) La recuperación de las referencias espaciales para comprender las unidades originarias de inhumación.

(b) La composición sexual y etaria de la serie bioantropológica.

(c) Una comparación regional de la estructura sexoedad con cementerios coetáneos.

Sobre la base de esos resultados, ofrecemos las primeras hipótesis respecto a la política sexual implementada en lo que puede haber constituido una profunda transformación en los mecanismos de reproducción social entre el periodo Arcaico y el Formativo.

\section{Representación Social y Prácticas Funerarias}

Señalar que el espacio funerario constituye una práctica de representación social no es una idea nueva y ha sido empleada en bioarqueología para comprender los filtros, normas culturales e ideologías que sostienen los órdenes simbólicos de la organización social humana (Castro-Martínez et al. 1995; Lull et al. 2005; Lull y Picazo 1989). Junto con otras formas de representación social que poseen una manifestación material, como la iconografía o los espacios destinados a prácticas políticas-ideológicas (Castro et al. 1996), el estudio de su variabilidad y estructuración contribuye al entendimiento de la valoración que ciertos colectivos poseen de algunos trabajos y de los sujetos sociales que los realizan, ocultando o sobrevalorando producciones que, o bien aparecen como relevantes entre las actividades económicas documentadas en el registro arqueológico, o son consustanciales al sostenimiento de la vida social. Un ejemplo del primer caso, podría ser la sobrerrepresentación de escenas de grandes cacerías en contextos agroganaderos (Escoriza Mateu 2006); mientras que en el segundo caso, se encuentran todos aquellos trabajos esenciales para la continuidad de la vida, como los procreativos, los de cuidado y los de mantenimiento de sujetos y objetos (Sanahuja 2007). Ya que una parte de dichos trabajos recaen en la potencia orgánica del cuerpo femenino, el estudio de los cementerios ofrece una multiplicidad de líneas de evidencia para acceder a la condición de los cuerpos, a la estructuración de los filtros culturales que definen el ingreso al espacio comunitario de la muerte y a la valoración de los difuntos, mediante el estudio de los trabajos destinados al espacio, el rito y conmemoración de la memoria de las personas inhumadas (Rihuete 2003).

En este trabajo nos concentramos en el estudio de la composición sexual y etaria de $\operatorname{Tr}-40$ y su comparación con la estructura de otros cementerios, con el objetivo de valorar las normas de ingreso al espacio comunitario de la muerte como una vía de análisis para pensar en las formas que articularon los mecanismos de cohesión y tensión social. Asimismo, la estructura sexo-edad ofrece elementos de juicio preliminares para proyectar hipótesis paleodemográficas orientadas a comprender la gestión social de los trabajos procreativos, y con ello, el control de la sexualidad de las personas y las relaciones estructurales de sexo (Mathieu 1971).

\section{La Gestión de los Trabajos Procreativos y la Reproducción Social}

El asunto de la procreación ha sido un hecho habitualmente concebido en su dimensión biológica, como si aquello le eximiera de regulación social. Han sido las feministas materialistas quienes realizaron una reconceptualización de estos trabajos, al destacar las relaciones sociales implicadas en la gestión del cuerpo reproductor y los agentes que intervienen en los diferentes momentos de la secuencia reproductiva (Tabet 2018:126). Efectivamente, el concepto demográfico de fertilidad natural, que define aquella fertilidad de las parejas o mujeres que no emplean métodos anti o contraceptivos para espaciar los nacimientos y el número de hijas e hijos, contrapuesto al de fertilidad controlada o regulación de la fertilidad, cuando se realizan intervenciones limitativas sobre los nacimientos, son entendidos por Paola Tabet (2018:127) como el resultado de una "ideología científica" que ha 
contribuido a suprimir del análisis de la reproducción, las relaciones de poder, las relaciones sociales entre hombres y mujeres y, en definitiva, a considerar la reproducción como un asunto de mujeres o como una peculiaridad de ellas.

Por el contrario, corrientes feministas desde la década de 1960, han propuesto una profunda transformación en el estudio social, económico, antropológico e histórico de la reproducción social, al incorporar el reconocimiento de los trabajos reproductivos y de cuidados como elementos centrales para la continuidad de la vida social, y destacar que su organización es materia de estudio (Carrasco 1991; Carrasco et al. 2019; Carrasquer 2013; Delphy 1987; Meillassoux 1982; Narotzky 1995; Sanahuja 2002, 2007; Tabet 2005).

Entre las propuestas más atractivas para la arqueología, se encuentra la que deriva de la pregunta acerca de cómo se asegura la coincidencia entre el momento de la ovulación y el apareamiento, en el entendido que el impulso sexual humano no se encuentra sincronizado con ese momento y tampoco es identificable con seguridad. La única vía para el logro exitoso de la fecundación es la regularidad y frecuencia de las relaciones sexuales, cuya institucionalización queda garantizada en la figura del matrimonio (Tabet 2018:132). De esta manera, el matrimonio constituye una alianza social que garantiza la exposición permanente al riesgo de embarazo. Para que ello sea posible, se requiere una domesticación de la sexualidad femenina, que es la domesticación de las mujeres a la reproducción. La pregunta que cabe, entonces, es cuándo se requiere una exposición permanente al riesgo de embarazo, es decir, en qué momento las sociedades reclaman más hijos e hijas.

La respuesta a esta pregunta la ofrece una numerosa bibliografía etnográfica e histórica que ha permitido teorizar en torno a las formas en que se organiza la gestión de estos trabajos para dar respuesta a transformaciones sociales y políticas.

En este trabajo recogemos buena parte de estas propuestas para entender, sobre la base de la evidencia arqueológica disponible, en qué momento o mediante qué procesos se pudo haber experimentado una transformación de la política sexual para responder a los nuevos escenarios económicos y sociales que configuran la reproducción social de la comunidad formativa tarapaqueña $y$, particularmente, de qué modo pudo afectar al control de la sexualidad de los cuerpos femeninos, al impacto en su capacidad para participar de la vida política pública y a la transformación de los órdenes simbólicos respecto a la maternidad y los trabajos de cuidado.

\section{La estructura sexo-edad y el problema sexo-género}

En arqueología y, en general, en la ciencia social normal (Kuhn 2006), suele existir cierto desconocimiento de los debates, antagonismos filosóficos y las agendas políticas de las diversas corrientes feministas. Un revisor de este artículo, por ejemplo, observó cierta incoherencia al referirnos a "mujeres" únicamente a través del cuerpo sexuado, al considerar que desarrollábamos una "perspectiva de género" en nuestra investigación y que, por lo tanto, estábamos estableciendo una identidad entre biología y, lo que podríamos definir como construcción cultural de la identidad psicosexual (Millet 2010). Aquello es válido para las perspectivas que se inspiran, básicamente, en la noción de "sistema sexo-género" propuesto por Ann Oakley en 1972 (Oakley 2015), que depende fuertemente de la dicotomía naturaleza-cultura (Tubert 2003).

Al contrario, en este trabajo recogemos el concepto de relaciones estructurales de sexo de Nicole-Claude Mathieu (1971), el cual, desde una perspectiva feminista socio-antropológica, más que psicoantropológica, se concentra en definir la condición social e histórica de las relaciones entre hombres y mujeres. A diferencia del sistema sexo-género, que establece una diferencia entre la biología del sexo y lo cultural del género en las mismas coordenadas ilustradas que persigue deshilvanar, la idea de las "relaciones estructurales", traducida del francés rapport social, describe relaciones abstractas entre grupos de personas o clases de personas, de manera diferente a los vínculos que se establecen en la relation social, que constituye una relación individual, cotidiana y concreta entre sujetos particulares, es decir, una relación interpersonal (Falquet 2018). Se trata de una perspectiva interseccional, en la que el foco se orienta a estudiar las relaciones y no los individuos ni sus relaciones particulares que, en todo caso, pueden derivar o no de las circunstancias estructurales que su contingencia histórica les obliga a experimentar.

Esta elección teórica proviene del interés político de nuestra investigación, la cual busca contribuir al conocimiento socio-antropológico de la variación de la organización social de los trabajos procreativos y de cuidados, en el entendido que han constituido históricamente un mecanismo estructural de explotación económica y de dominación política de las mujeres. Si bien esta perspectiva no es disonante con preguntas acerca del estudio de la identidad psico-sexual en bioarqueología, 
constituyen caminos que deben recurrir a líneas de evidencias y formulaciones conceptuales específicas.

\section{El Cementerio Tarapacá 40}

El cementerio Tarapacá 40 (Tr-40), ubicado en la porción baja de la quebrada homónima (1958'40”S, $69^{\circ} 33$ '52" W, Figura 1), posee una cronometría que le sitúa entre los años $1110 \mathrm{cal}$ AC-660 cal DC (Uribe et al. 2015). A partir de su estudio ha sido posible conocer algunos aspectos centrales de las sociedades que habitaron la porción baja de la quebrada, como sus prácticas funerarias, condiciones de salud y relaciones regionales. El sitio representa un espacio de uso funerario que ha sido entendido como la expresión mortuoria de la población que habitó los asentamientos de Pircas y Caserones (Núñez 1982, Uribe et al. 2015) (Figura 1B). Tanto su configuración espacial, demarcada por postes de algarrobo, como la disposición, profundidad y composición de los bienes funerarios que acompañaron a los entierros, localizan a Tr-40 dentro una tradición formativa de raigambre arcaica costera. Destaca el uso de turbantes, el enfardado en mantas y pieles de animales con amarras en fibras vegetales para la preservación y transporte de los cuerpos, el uso de grandes cestos realizados en técnica de espiral para depositar y tapar los fardos, junto con un abundante y diverso repertorio de bienes funerarios. Entre ellos, sobresalen objetos para labores de caza, cestería, textilería, labrado, trabajo forestal y molienda, así como múltiples alimentos procedentes del interior y la costa, además de objetos de uso personal como penachos de plumas, collares, pendientes, tobilleras, sandalias de cuero, bolsas, etc.

Este cementerio fue excavado arqueológicamente en la década de 1960 bajo un proyecto de colaboración entre la Universidad de Chile y la Universidad de California, a cargo de Lautaro Núñez y Delbert True (Núñez 1969, 1982; True 1980). De las campañas realizadas entre 1966 y 1967 deriva la mayor cantidad de material arqueológico conocida (Núñez 1969, 1970, 1972, 1974, 1976, 1982, 2006; True 1980),

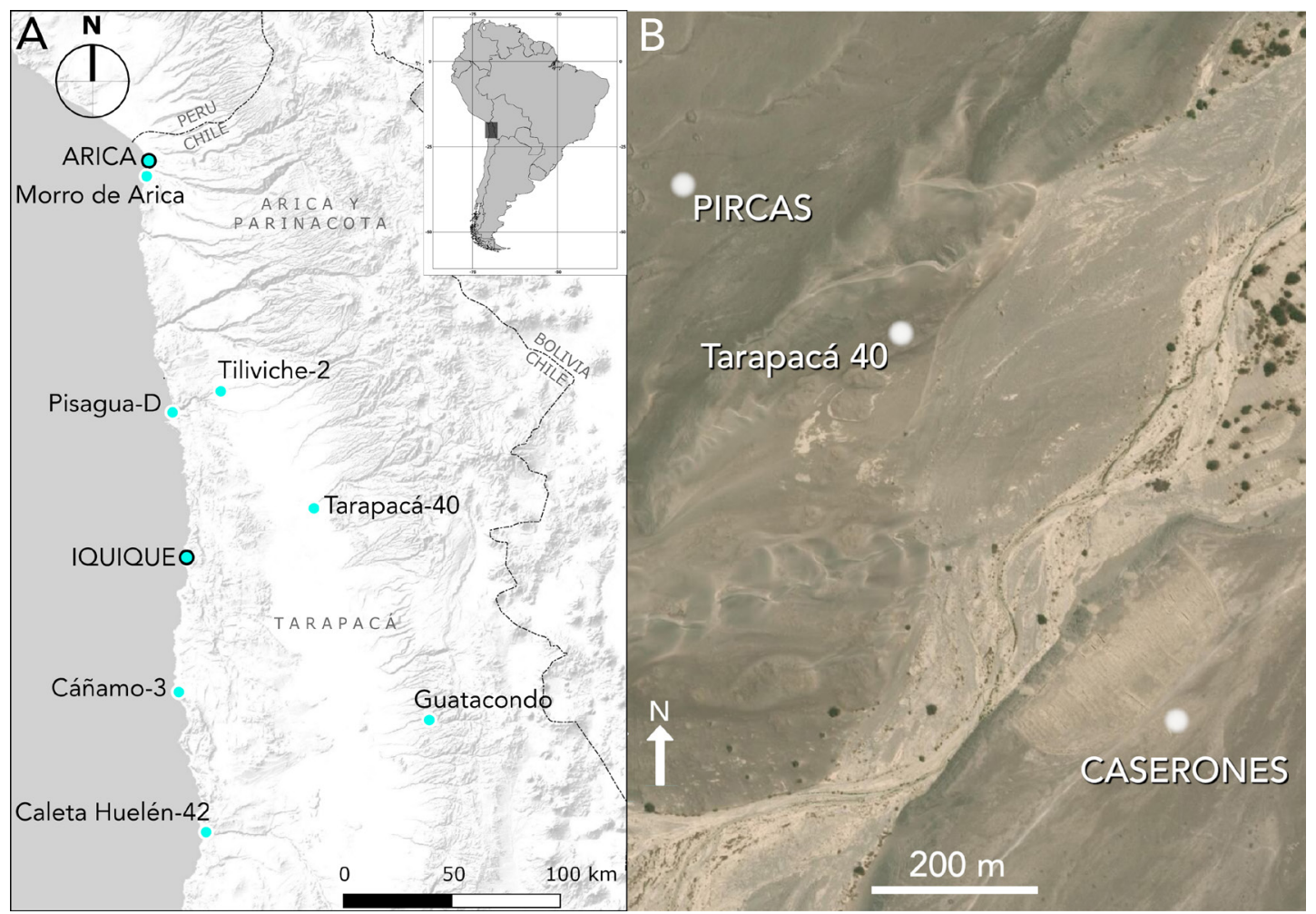

Figura 1. (A) Mapa con la ubicación de los principales sitios arqueológicos mencionados en este artículo. (B) Sección inferior de la quebrada de Tarapacá con la ubicación del cementerio Tarapacá 40 y sus asentamientos asociados.

(A) Map outlining the location of the main archaeological sites mentioned in article. (B) Lower section of the Tarapaca gorge with the location of the Tarapacá 40 cemetery and its associated settlements. 
mientras que una porción menor proviene de una pequeña intervención realizada entre 1980 y 1981 (Núñez 1982). Se tienen noticias de dos excavaciones adicionales muy puntuales y restringidas que no forman parte de las colecciones consultadas en este trabajo (Méndez-Quirós 2009).

La mayoría de los materiales arqueológicos obtenidos durante las excavaciones principales, fueron albergados por diversas instituciones del norte de Chile, hasta que en la década de 1990 se almacenaron en las dependencias de la Estación Experimental de Canchones de la Universidad Arturo Prat bajo la tutela del Profesor Dr. Álvaro Carevic, donde luego fueron inventariados gracias a un Proyecto FONDART que lideró el arqueólogo Pablo Méndez-Quirós (2009).

Otra parte del material arqueológico proveniente de $\operatorname{Tr}-40$ se encuentra en diversas instituciones nacionales e internacionales, como el Museo Arqueológico San Miguel de Azapa (MASMA), el Museo Regional de Iquique, la Universidad de Antofagasta, la Universidad de Chile, la Comunidad de Huarasiña y la Universidad de California. Sin embargo, no todas las instituciones cuentan con un inventario actualizado, de modo que el universo final de material recuperado durante las excavaciones del cementerio aún es incierto.

Tampoco se ha publicado una descripción sistemática de las excavaciones, ni se conoce la planimetría del cementerio, sino solo referencias parciales de las actividades realizadas. Gracias a esas referencias sabemos que se habrían exhumado una cantidad superior a 110 cuerpos; en su mayoría de un sector denominado Tr-40A, señalado como más temprano (Agüero 2012; Núñez 1970, 1981, 1982, 2006; Núñez y Santoro 2011; Uribe et al. 2015). No obstante, la referencia a tumbas, cuerpos, individuos o registros como sinónimos, ha generado una gran confusión al obviarse la existencia de entierros colectivos o elementos óseos aislados provenientes de eventos no funerarios o secundarios. En este sentido, se ha asumido como cierta una supuesta estructuración cronológico-espacial del cementerio (Sectores A y B), a pesar que su detalle se desconoce totalmente.

La única información publicada que ofrece nociones respecto a algunos elementos de la organización espacial del cementerio y de los criterios arqueológicos empleados para registrarla, es una transcripción realizada de una parte del cuaderno de campo de Lautaro Núñez en la publicación de Uribe et al. (2015:Nota 1). Esta señala que el Cementerio Tr-40 se organiza en sectores (A-B), secciones (A-Z,
A’-Z') y tumbas (numeración arábica, correlativa y finita por sección). Los sectores estarían segregados espacialmente, pero no se conoce información respecto de la cantidad y cualidad de su discontinuidad física (Figura 2). Por su parte, la letra reflejaría el orden de su intervención arqueológica, siendo el denominado $\mathrm{A}$, un sector localizado al este y primero en ser excavado; y B, un sector identificado al oeste del primero e intervenido con posterioridad (Oakland 2000:Figura 12.3, Uribe et al. 2015:Nota 1).

Los sectores habrían sido subdivididos en secciones que, según las descripciones del cuaderno de campo de L. Núñez (Uribe et al. 2015:Nota 1), podrían definirse como "fajas" o "franjas" paralelas separadas un metro de ancho. No obstante, no se menciona la distribución de las secciones por Sector, de modo que se desconoce el ordenamiento espacial de las secciones y tumbas. Por otra parte, hay contradicciones en la atribución de sector de las secciones. Por ejemplo, se menciona que la sección M' pertenece al Sector A, pero también se señala que las secciones con letra prima habrían sido empleadas para realizar una ampliación de las franjas en el Sector B.

Por lo tanto, la única referencia espacial que sostendría relativa confiabilidad, por el momento, es la que establece una relación sección-tumba, es decir, letra mayúscula-número, pero no su relación con el sector.

\section{La serie bioantropológica del Cementerio Tr-40}

La mayor parte de los materiales bioantropológicos fueron trasladados, pocos años después de su obtención, al campus Macul de la Universidad de Chile. Desde entonces, y al igual que otras colecciones generadas a partir de 1950, han experimentado diversos procesos de manipulación, traslado, deterioro y recuperación (Lemp et al. 2008).

Los primeros estudios tuvieron un enfoque paleopatológico, especialmente orientado a la información que ofrecen los tejidos blandos, abundantes en un primer momento (Allison et al. 1981; Allison et al. 1979; De Araújo et al. 1985; Fontana et al. 1983; Munizaga et al. 1978a; 1978b; Rothhammer et al. 1984; 1985). Destacan los estudios de dieta a través del análisis de contenido intestinal (Holden y Núñez 1993) y, posteriormente, los isotópicos (Santana-Sagredo et al. 2015, Santana et al. 2012); junto con aquellos sobre modos de vida mediante evaluación osteológica (Herrera 2010).

La osteobiografía de 51 cuerpos, se logró conocer recién en 2010 (Herrera 2010). En este estudio se 


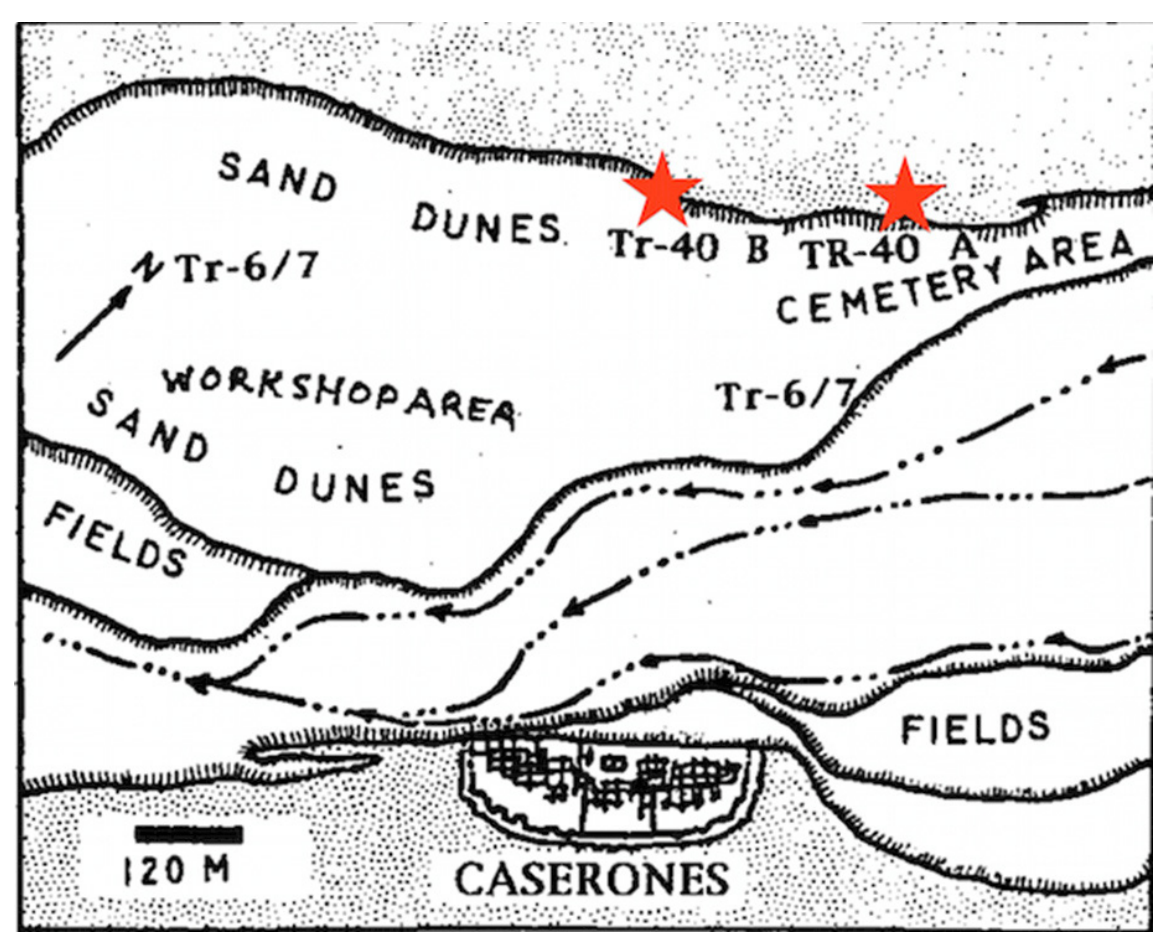

Figura 2. Posición relativa de los sectores A y B del cementerio Tarapacá 40, según Oakland (2000:Figura 12.3). Se ha modificado el original destacando su posición con estrellas rojas.

Relative position of sectors $A$ and $B$ of the Tarapacá 40 cemetery, according to Oakland (2002: Figure 12.3). The original has been modified, using red starts to highlighting its position.

señala que los "esqueletos de este cementerio (...) se hallan incompletos, con pérdidas óseas importantes, como son coxales y costillas, además, de presentar muchos de ellos solo algunas piezas óseas (cráneos o huesos largos)" (Herrera 2010:10). Efectivamente, un conjunto de material bioantropológico se encontraba aún almacenado en contenedores ${ }^{1}$, sin preparar ni conservar hasta el año 2008 cuando se iniciaron acciones de restitución de los 51 cuerpos que fueron sometidos a "limpieza mecánica, conservación y rotulado" (Herrera 2010:9).

Hasta ese momento, solo el estudio de Herrera había considerado las asociaciones contextuales arqueológicas (Herrera 2010; Uribe et al. 2015), a diferencia de los trabajos sobre tejidos blandos, los cuales fueron empleados en su mayoría para estudios comparativos que no las consideraron. Por lo tanto, no se sabe qué cuerpos fueron los muestreados, de manera que si bien tenemos conocimiento de tuberculosis, neumonía o Chagas en la población de $\operatorname{Tr}-40$, desconocemos quienes fueron las personas enfermas, sus características y sus asociaciones.

No obstante el esfuerzo y relevancia del trabajo de Herrera (2010), en los almacenes de la Facultad de
Ciencias Sociales de la Universidad de Chile (FACSO) permaneció una cantidad voluminosa e indeterminada de material bioantropológico dispuesto en cajas de cartón y bolsas plásticas. Buena parte mantenía porciones momificadas y materiales arqueológicos asociados, especialmente textiles de fardo y vestimenta, objetos personales de atuendo y ajuar como turbantes, cuentas de collar, tobilleras y puntas de proyectil. Además, varios fardos conteniendo restos de infantes se encontraban aún completos. El deterioro activo por años de condiciones ambientales y de manejo inadecuadas era visible en la biocolonización por hongos e insectos, desmembramiento y fragmentación, tanto en tejidos humanos como en piezas arqueológicas. Entre los daños que generaban más preocupación, estaba el sufrido por las referencias de origen del material, las que, en forma de etiquetas en papel o cartón, se hallaban en proceso de desintegración. En algunos casos, los restos presentaban esta información cifrada sobre la superficie de algunas piezas óseas o en etiquetas de cartón sujetas a los fardos mediante alambres oxidados y frágiles. Mientras unas eran parcial o totalmente ilegibles, otras directamente se habían perdido o bien habían experimentado mutaciones al ser replicadas. 
La última sistematización y ordenamiento de la colección fue posible, a partir del año 2016, gracias a una colaboración entre el Proyecto Piloto de Conservación Bicentenario de la Unidad de Conservación de FACSO y el Proyecto FONDECYT de Postdoctorado 31620759 (González-Ramírez 2019). Como parte de las actividades dedicadas a la colección bioantropológica, se confirmaron las apreciaciones de Herrera respecto a su parcialidad, mientras que se despejó la incertidumbre respecto de su calidad informativa, a través de la cuantificación individual y colectiva de los estados de preservación y momificación de la totalidad del conjunto (Sáez y González-Ramírez 2018). Asimismo, las referencias espaciales aún presentes en el material almacenado en FACSO y Canchones fueron examinadas con el fin de reconstruir e integrar las asociaciones arqueológicas, puestas en riesgo por la separación de la colección general y los estragos causados por el traslado forzoso al que fueron sometidas en dictadura. Los resultados de esta labor aplicada a la serie bioantropológica de la Colección Tr-40 son presentados aquí2.

\section{Material y Metodología}

En primer lugar, se realizó un examen visual directo, registro escrito y fotográfico del rotulado de materiales bioantropológicos y sus contenedores, con el fin de resguardar su información espacial y contextual, respecto a las asociaciones de Sección y Tumba.

Considerando la alta diversidad en la preservación de cuerpos y segmentos corporales (Sáez y GonzálezRamírez 2018), la determinación de sexo y estimación de edad se realizó sobre un amplio conjunto de indicadores de su variación en cuanto a propiedades de forma, tamaño y textura de piezas óseas y su superficie cortical. Tal variación debe ser atribuida, por una parte, a la expresión del dimorfismo sexual en la morfología esqueletal, y por otra, a los procesos de crecimiento, desarrollo y los cambios degenerativos vinculados en distinta magnitud al envejecimiento.

Para la determinación de sexo se aplicó el cálculo del grado de sexualización de los indicadores dimórficos para pelvis y cráneo (Ferembach et al. 1980), el cálculo de índice isquiopúbico (Washburn 1948), índice sacro (Kimura 1982), triángulo mastoidal (Retamal y Sáez 2006; Sáez y Manríquez 2006) y el método de Phenice (1969). Además, se empleó un conjunto de funciones discriminantes obtenidas de poblaciones guatemaltecas, indias y chilenas para longitudes y anchos de huesos largos (Purkait 2003;
Purkait y Chandra 2004; Ríos Frutos 2002, 2003a, 2003b, 2005; Ross y Manneschi 2011).

En adultos la estimación de edad se realizó mediante la observación y registro de las modificaciones degenerativas de la sínfisis púbica (Brooks y Suchey 1990; Todd 1920), superficie auricular (Lovejoy et al. 1985), cuarta costilla (Iscan et al. 1984); secuencia de cierre de suturas craneales (Meindl y Lovejoy 1985), suturas palatinas (Mann et al. 1991) y desgaste dental (Brothwell 1981; Lovejoy 1985; Miles 1963). En aquellos cuerpos pobremente preservados o fragmentarios, en ausencia de otros datos de mejor calidad y considerando la relación progresiva entre el proceso degenerativo esqueletal, los fenómenos osteoartrósicos y la edad, se estimó el desarrollo de indicadores de enfermedad articular degenerativa, según valores leve, moderado y severo. Se estableció una edad de 30 años para los cuerpos con desarrollo leve de indicadores, 40 años para aquellos con desarrollo moderado y 50 años para el desarrollo intenso de los indicadores artropáticos (Anderson y Loeser 2010).

En cuerpos no-adultos, la edad fue estimada mediante la observación del desarrollo de las piezas dentarias (Smith 1991), del hueso timpánico (Weaver 1979), el cálculo de ecuaciones de regresión sobre la longitud de la diáfisis de huesos largos, isquion y pubis (Cardoso et al. 2014a; Primeau et al. 2016; Rissech y Black 2007; Rissech et al. 2003; Rissech et al. 2013a; Rissech y Malgosa 2007; Rissech et al. 2013b), porción basilar del occipital (Irurita y Aleman 2017) y la secuencia de fusión de los centros de osificación primarios y secundarios (Bajwa et al. 2013; Becker 1986; Cardoso 2008a, 2008b; Cardoso et al. 2013; Cardoso et al. 2014b; Cardoso y Ríos Frutos 2011; Scheuer y Black 2000).

Los estadios definidos por Bogin y Smith (1996) fueron modificados, con el fin de simplificar la descripción, desde cinco a cuatro grupos etarios: infante (34 semanas gestacionales -36 meses), niño/a (agregado de los grupos niño y juvenil: 3-11,9 años), adolescente (12-19.9 años) y adulto (20 y más años) ${ }^{3}$.

La momificación presente en cerca de un tercio de los cuerpos comprometió la observación a ojo desnudo de una porción considerable de indicadores. Esta limitación fue superada en gran parte gracias a las imágenes de rayos $\mathrm{X}$ realizadas por los investigadores del grupo OSTeam (González-Ramírez et al. 2019).

Para evaluar comparativamente la estructura sexual y etaria de la serie bioantropológica de $\mathrm{Tr}-40$, consideramos las frecuencias de individuos no-adultos, 
mujeres adultas y hombres adultos provenientes de seis cementerios de la región adscritos a los periodos Arcaico y Formativo; las que fueron sometidas a un análisis de correspondencias simples con el propósito de identificar asociaciones entre los sitios atribuibles a esa estructura sexo-edad. Los análisis y test de significancia estadística fueron realizados con el software RStudio 1.2.5033 (R Core Team 2019) y el paquete FactoMineR (Lê et al. 2008).

A diferencia de los grupos etarios establecidos para el estudio interno de Tr-40, en esta evaluación se debió definir únicamente dos grandes grupos que recogieran la diversidad clasificatoria ofrecida en los trabajos de los otros cementerios. De esta manera, el límite entre adultos y no-adultos fue establecido en los 15 años, lo que sostiene cierta coherencia con la edad descrita para el inicio social de la actividad sexual y la consolidación laboral en varios lugares y épocas de la geografía andina (Arnold 1997; Carrasco y Gavilán 2009; Dean 2001).

Aunque los trabajos que ofrecen esta clase de datos no suelen aportar antecedentes respecto a la sistematicidad de las excavaciones arqueológicas, al manejo de las colecciones de los materiales publicados ni a los métodos específicos utilizados en el diagnóstico de sexo y edad, asumimos provisoriamente que los casos informados son representativos de la totalidad de los cementerios en cuestión.

\section{Resultados}

\section{Referencia espacial y unidades de asociación contextual}

Se reconoció un total de 149 cuerpos, de los cuales un conjunto de 145 (97\%) se halla almacenado en FACSO, mientras que cuatro de ellos (3\%) se encuentran en la estación Canchones de la UNAP.

La referencia espacial de 135 cuerpos (91\%), distribuidos en nueve secciones, fue recuperada satisfactoriamente. Además, fueron identificados los restos de dos individuos recuperados de unidades de excavación no descritas previamente en los antecedentes de Tr-40 (Corte B y Sondeos) (Figura 3). Estos 135 cuerpos provienen de 102 tumbas, 22 de ellas colectivas, que contienen los restos de 55 individuos. En su mayoría $(\mathrm{n}=80)$, las tumbas fueron excavadas en la sección M', de las cuales se recuperaron 104 cuerpos, correspondiente al $70 \%$ de la serie esqueletal. La tumba 2 de la sección M' contiene la mayor cantidad de cuerpos, con seis individuos. El resto de secciones están integradas por no más de siete cuerpos, mientras que un $10 \%$ de los materiales $(n=14)$ no posee referencia a ninguna unidad de asociación arqueológica. Esta ausencia de información afecta especialmente a

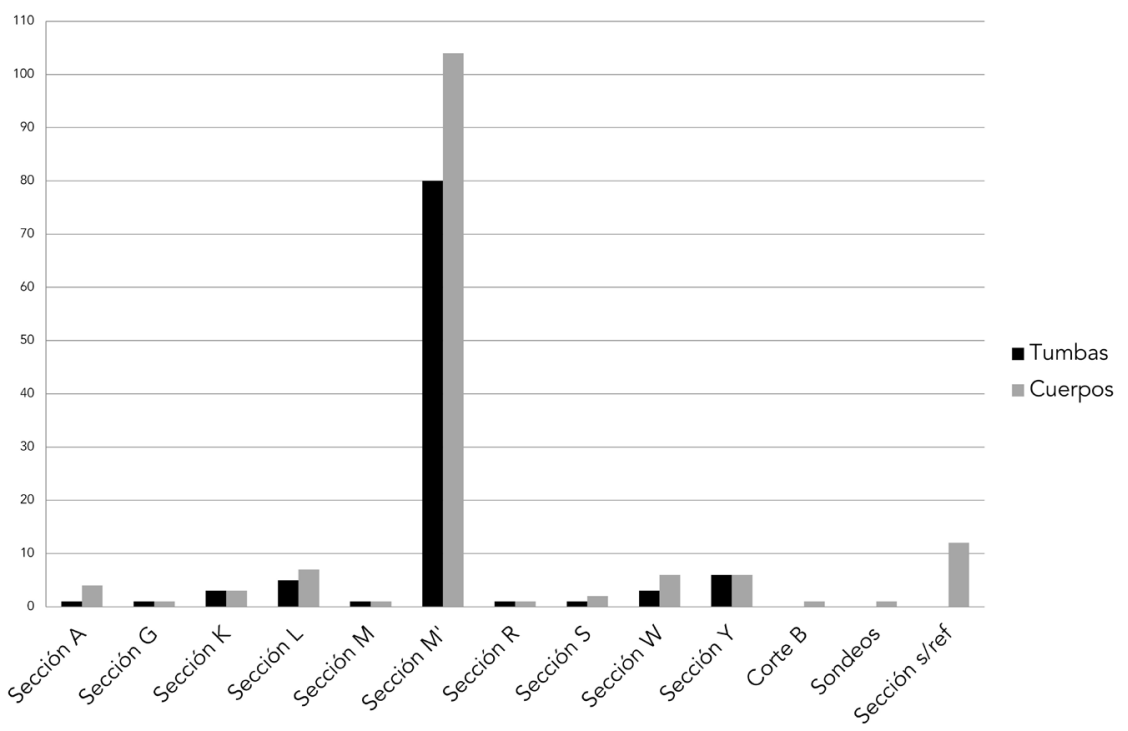

Figura 3. Frecuencia de tumbas y cuerpos examinados en $\mathrm{Tr}-40$ de acuerdo a su referencia espacial. Frequency of graves and bodies examined in Tr-40 according to their spatial reference. 
niñas/os e infantes, cuya representación en el grupo sin referencia (67\%) es casi el doble de la observada en la serie total (37\%).

\section{Sexo y Edad}

Los resultados del diagnóstico de sexo y edad para los 149 cuerpos de la serie de Tr-40 se hallan resumidos en la Tabla 1. Todos los grupos etarios se encuentran representados en la colección de Tr-40. El 56\% de los cuerpos pertenecen a individuos de edad adulta. De estos, el $97,6 \%$ de los cuerpos fue identificado efectivamente como femenino $(51,5 \%)$ o masculino $(48,4 \%)$, mientras que los cuerpos de sexo indeterminado corresponden a un 35\% del total de la colección.

\section{La estructura sexo-edad de $\operatorname{Tr}$-40 en el contexto regional}

La Figura 4 y la Tabla 2 muestran las frecuencias relativas de las variables sexo-edad consideradas para los seis cementerios incluidos en esta comparación. Se observa una relación inversa entre mujeres adultas y no-adultos la cual fue confirmada mediante un test de correlación que arrojó una relación fuerte $(r=-0,9 /$
Tabla 1. Distribución sexual y etaria de la serie bioantropológica del cementerio $\operatorname{Tr}-40$. Sexual and age distribution for Tr-40 bioanthropological series.

\begin{tabular}{lccccc}
\hline & Fem & Masc & Indet & Total & $\%$ \\
\hline Infante & 0 & 0 & 35 & 35 & 23 \\
Niño & 1 & 4 & 14 & 19 & 13 \\
Juvenil & 3 & 5 & 3 & 11 & 7 \\
Adulto & 45 & 37 & 2 & 84 & 56 \\
Total & 49 & 46 & 54 & 149 & \\
\hline
\end{tabular}

$\mathrm{p}=0,008)$. Esto no se replica entre ellas y los adultos masculinos, ni entre ellos y los individuos no-adultos.

Un análisis de correspondencias simples muestra una estructuración dicotómica (Figura 5): por una parte, sitios costeros con una alta frecuencia de mujeres de edad adulta (Grupo 1) y, por otra, cementerios del interior y uno costero (Cáñamo 3), con frecuencias femeninas menores y presencia elevada de no-adultos, en una proporción cercana a los tres tercios (Grupo 2). La elevada explicación de la varianza en la primera dimensión $(89,6 \%)$, admitió la realización del test ANOVA que confirmó que las diferencias observadas entre ambas agrupaciones son estadísticamente significativas $(\mathrm{p}=0,005)$.

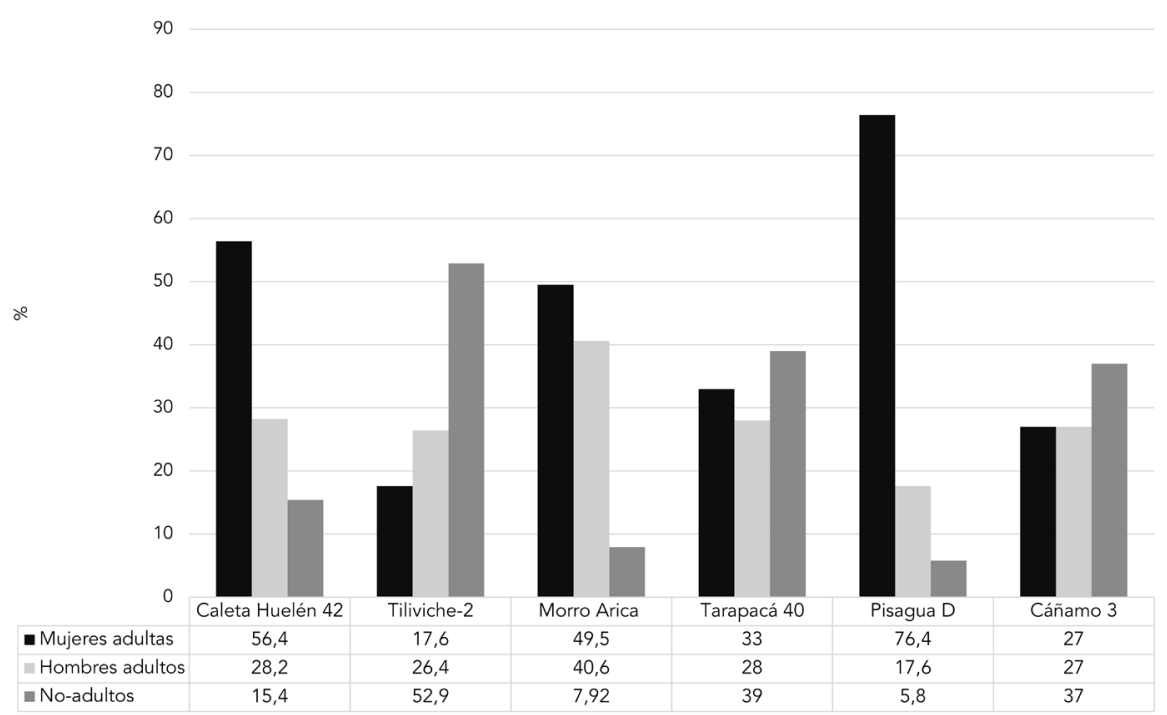

Figura 4. Gráfico de frecuencias relativas de individuos adultos (hombres y mujeres) y no-adultos de algunos cementerios de la transición arcaico-formativa y periodo Formativo del extremo Norte de Chile. Graph of relative frequencies of adult individuals (men and women) and non-adults of some cemeteries of the archaic-formative transition and Formative period of the extreme North of Chile. 
Tabla 2. Frecuencias y porcentajes de adultos y no-adultos en cementerios de la transición arcaico-formativa y periodo Formativo del extremo Norte de Chile. Los casos que no completan 100\% corresponden a pequeñas fracciones de adultos de sexo indeterminado que no han sido incluidos en esta comparación.

Frequency of adults and non-adults in cemeteries from the archaic-formative transition and Formative period of the extreme north of Chile. Cases that do not reach 100\% correspond to small fractions of undetermined sex adults not included in this comparison.

\begin{tabular}{|c|c|c|c|c|c|}
\hline Cementerio & $\begin{array}{c}\text { Fechas } \\
(\mathrm{SHCal} 13-2 \sigma)\end{array}$ & $\begin{array}{l}\text { Mujeres } \\
\text { adultas }\end{array}$ & $\begin{array}{l}\text { Hombres } \\
\text { adultos }\end{array}$ & $\begin{array}{l}\text { No-adultos } \\
\quad<15\end{array}$ & Referencias \\
\hline $\begin{array}{l}\text { Caleta Huelén } 42 \\
\text { (costa) }\end{array}$ & $\begin{array}{l}3762-3125 \mathrm{cal} \mathrm{AC} \\
2458-1924 \mathrm{cal} \text { AC }\end{array}$ & $\begin{array}{l}22 \\
56,4 \%\end{array}$ & $\begin{array}{l}11 \\
28,2 \%\end{array}$ & $\begin{array}{l}6 \\
15,4 \%\end{array}$ & Cocilovo et al. 2005; Núñez 1976 \\
\hline $\begin{array}{l}\text { Tiliviche-2 } \\
\text { (interior) }\end{array}$ & 2462-1901 cal AC & $\begin{array}{l}6 \\
17,6 \%\end{array}$ & $\begin{array}{l}9 \\
26,4 \%\end{array}$ & $\begin{array}{l}18 \\
52,9 \%\end{array}$ & Standen y Núñez 1984 \\
\hline $\begin{array}{l}\text { Morro de Arica } \\
\text { (costa) }\end{array}$ & $\begin{array}{l}1661-1527 \mathrm{cal} \mathrm{AC} \\
577-632 \mathrm{cal} \text { DC }\end{array}$ & $\begin{array}{l}50 \\
49,5 \%\end{array}$ & $\begin{array}{l}41 \\
40,6 \%\end{array}$ & $\begin{array}{l}8 \\
7,92 \%\end{array}$ & Costa et al. 2000 \\
\hline $\begin{array}{l}\text { Tarapacá } 40 \\
\text { (interior) }\end{array}$ & $\begin{array}{l}1041-843 \mathrm{cal} \text { AC } \\
604-765 \mathrm{cal} \text { DC }\end{array}$ & $\begin{array}{l}49 \\
33 \%\end{array}$ & $\begin{array}{l}41 \\
28 \%\end{array}$ & $\begin{array}{l}57 \\
39 \%\end{array}$ & Sáez y González-Ramírez 2018; Uribe et al. 2015 \\
\hline $\begin{array}{l}\text { Pisagua D } \\
\text { (costa) }\end{array}$ & $\begin{array}{l}809-552 \mathrm{cal} \mathrm{AC} \\
803-546 \mathrm{cal} \text { AC }\end{array}$ & $\begin{array}{l}13 \\
76,4 \%\end{array}$ & $\begin{array}{l}3 \\
17,6 \%\end{array}$ & $\begin{array}{l}1 \\
5,8 \%\end{array}$ & Cocilovo et al. 1999 \\
\hline $\begin{array}{l}\text { Cáñamo } 3 \\
\text { (costa) }\end{array}$ & $\begin{array}{l}362-475 \mathrm{cal} \text { DC } \\
694-1020 \mathrm{cal} \text { DC }\end{array}$ & $\begin{array}{l}6 \\
27 \%\end{array}$ & $\begin{array}{l}6 \\
27 \%\end{array}$ & $\begin{array}{l}8 \\
37 \%\end{array}$ & $\begin{array}{l}\text { Arias 2010; Arias y Herrera 2012; González-Ramírez } \\
\text { 2019; González et al. 2017; Núñez y Moragas } 1977\end{array}$ \\
\hline
\end{tabular}

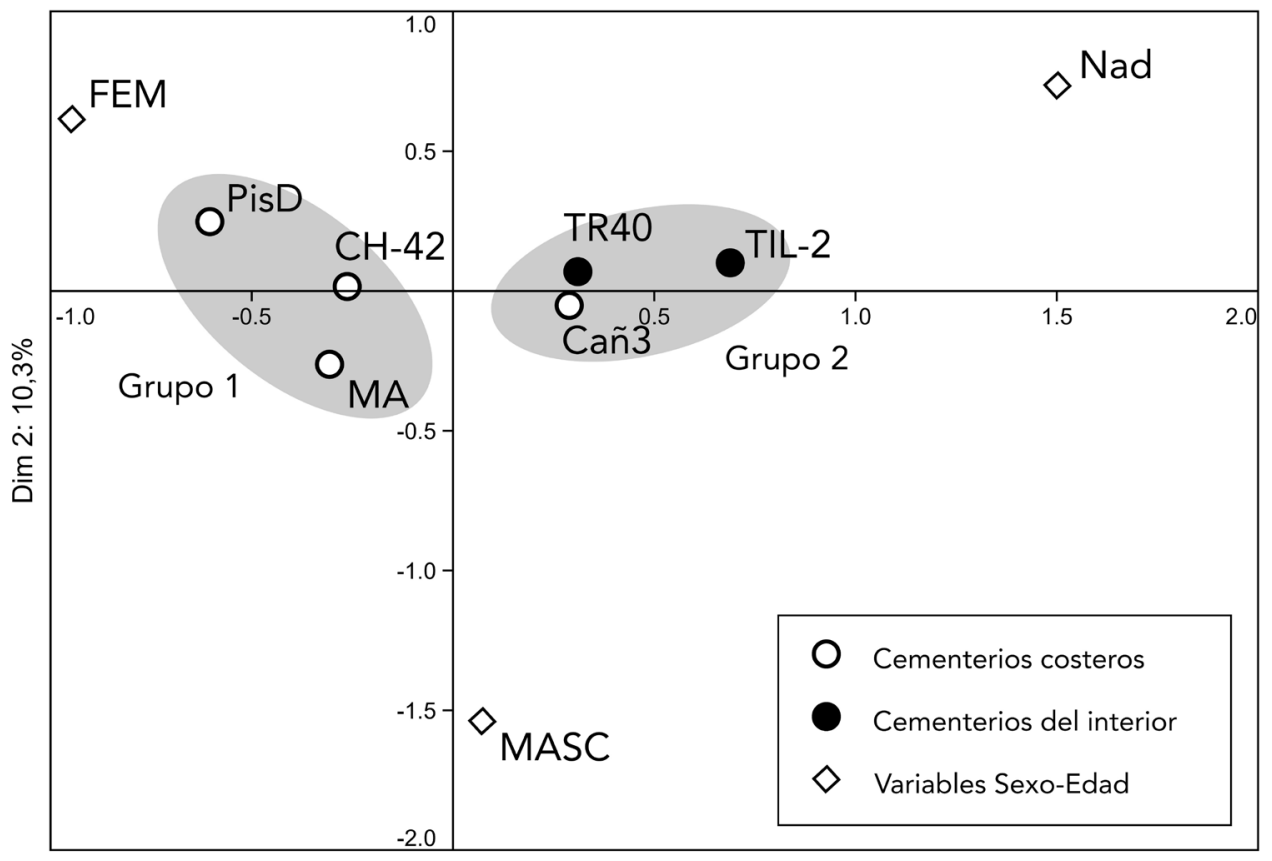

Dim 1: $89,6 \%$

Figura 5. Análisis de correspondencias simples sobre las frecuencias relativas de individuos adultos masculinos (MASC), individuos femeninos adultos (FEM) e individuos no-adultos (Nad) de cementerios de la transición arcaico-formativa y periodo Formativo del extremo norte de Chile. CH-42: Caleta Huelén 42, TIL-2: Tiliviche-2, MA: Morro de Arica, TR40: Tarapacá 40, PisD: Pisagua D, Cañ3: Cáñamo 3.

Simple correspondence analysis on relative frequencies of adult male and female bodies and non-adult bodies of the archaic-formative transition and Formative period cemeteries from extreme North of Chile. CH-42: Caleta Huelén 42, TIL-2: Tiliviche-2, MA: Morro de Arica, TR40: Tarapacá 40, PisD: Pisagua D, Cañ3: Cáñamo 3. 


\section{Discusión}

Los 149 individuos informados en este trabajo triplican el conocimiento bioantropológico que se contaba para este cementerio, enriqueciendo las bases empíricas para la comprensión de asuntos poblacionales, sociológicos y antropológicos del periodo Formativo de la Quebrada de Tarapacá.

El número de cuerpos que ha perdido su referencia de asociación arqueológica puede ser considerado como bajo $(n=14,9 \%)$. Este valor sugiere que los responsables de la tutela de la colección, si bien no contaron con las condiciones idóneas para su manejo, entendieron la relevancia del resguardo de la información contextual como fuente de información científica (Lemp et al. 2008). De la conservación de dicha información se derivan dos consecuencias. La primera, es que la alta proporción de la Sección M' (70\%) concuerda con lo descrito en la literatura disponible (Figura 3), la cual señala una cantidad cercana a 100 unidades (Agüero 2012; Núñez 1982, 2006). La sumatoria de las secciones restantes, sin considerar los registros sin referencia ni los sondeos, alcanza a 22 tumbas y 31 cuerpos, lo que parcialmente coincide con las 16 tumbas recuperadas según L. Núñez en el Sector B (Núñez 1982). Si esto fuera correcto, podría sugerirse que las secciones noM'/M, corresponden al área descrita como Sector B. Sin embargo, esta idea no debería considerarse definitiva.

Una segunda consecuencia deriva de la distribución de los cuerpos según tumbas individuales y colectivas. Debido a la inexistencia de un catastro publicado del sitio y las tumbas excavadas, se desconocía el comportamiento de esta práctica funeraria en $\mathrm{Tr}-40$. Arqueológicamente, este hecho es significativo. Recordemos que la mayor parte de los bienes funerarios de las tumbas fueron separados de sus estructuras de inhumación y del o los cuerpos a los que se asociaron, cuando se envió el material bioantropológico a la Universidad de Chile en la década de 1970. La existencia de fardos en la colección Juan Munizaga, hace suponer que algunos llegaron en esa condición, pero la publicación de registros fotográficos de procesos de desenfardado in situ (p.ej., Núñez y Santoro 2011), indica probablemente, que los cuerpos llegaron a Santiago en un estado de desenfardado desigual y, hasta ahora, indeterminado. Por su parte, los bienes funerarios que quedaron en las colecciones nortinas, solo hacen referencia a "tumbas". Por lo tanto, la constatación de tumbas colectivas y la disgregación de los contextos de inhumación, plantea dificultades en la reintegración de las asociaciones originales, las cuales deberían ser tomadas en cuenta en futuros trabajos arqueológicos del cementerio $\mathrm{Tr}-40$. Con todo, las complejidades culturales y cronológicas que se desprenden de la estructura de asociaciones entre bienes, individuo y grupo en las estructuras colectivas de inhumación, plantean problemas de investigación muy sugerentes, especialmente, en relación a algunas prácticas funerarias documentadas entre las poblaciones andinas, entre las cuales, la revisita, el reacomodo, la intervención y la re-ritualización, constituyeron actividades recurrentes que hemos constatado en nuestra investigación (Figura 6), y que problematizan el análisis arqueológico del cementerio.

No obstante el valor de los resultados obtenidos, es necesario tomar algunos elementos en consideración respecto a la composición e integridad de la muestra. Por ejemplo, los 149 cuerpos restituidos por este trabajo representan un $16,2 \%(n=25)$ adicional que los informados en la literatura. Si bien no tenemos claridad si esta diferencia se debe a un error de inventario en la excavación, a un problema respecto a los casos informados en la literatura o a una sobrerrepresentación del número mínimo de individuos realizado en nuestro análisis, sospechamos que todos estos factores podrían concurrir en su conformación.

Por una parte, que el cementerio haya sido descrito empleando como sinónimos los términos tumba, cuerpo, inhumaciones y registros, constituye una primera condición que favorece la confusión y la ambigüedad. En este sentido, que no se conoce el control de los materiales durante la excavación y el catastro definitivo de los cuerpos recuperados, podemos sugerir que la confusión entre los términos empleados, plantea eventuales incongruencias respecto a la caracterización del contexto funerario y al manejo del inventario durante la excavación.

Por otra parte, si bien se menciona la presencia de tumbas colectivas (Núñez 1984), no tenemos noticias respecto a su distribución, composición ni procedencia espacial. De acuerdo a la información obtenida de la literatura acerca del número de registros (Tabla 3), hemos constatado que la pérdida de información contextual ha afectado, fundamentalmente, a cuerpos de individuos infantiles y niños. Este hecho sugiere que la discrepancia en estos 25 cuerpos puede deberse no solo a una pérdida de la información original, sino también, a la historia de los traslados y el desinterés en las preguntas de investigación por algunos grupos etarios.

Finalmente, es importante mencionar que parte del incremento del número total podría ser explicado 

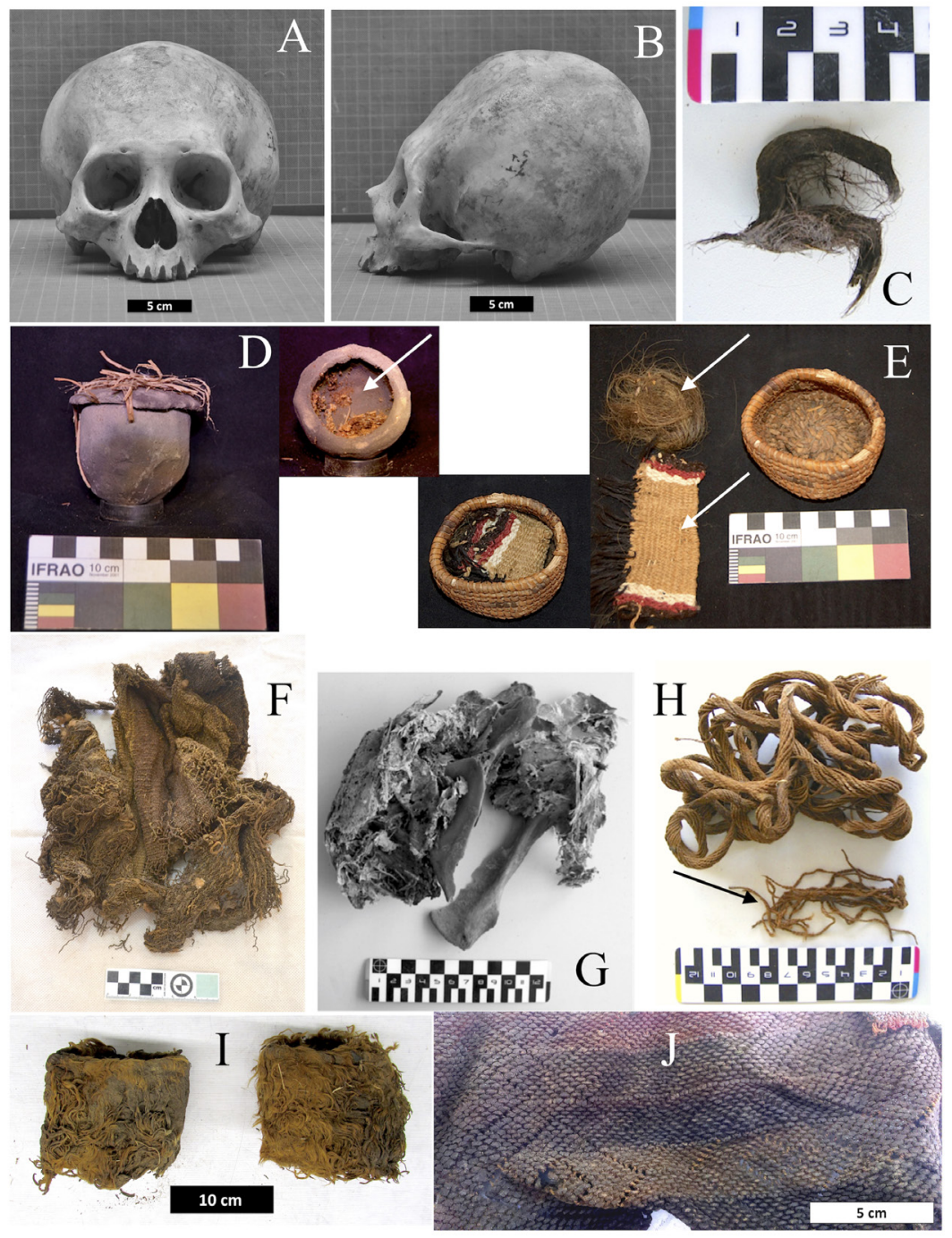

Figura 6. Muestra de materiales de la Tumba 1 Sección L Tr-40. (A y B) Frontal y lateral cráneo ID016, maculino-17 años, Inventario $\mathrm{N}^{\circ}$ B2138 FACSO, 370-205 cal AC, D-AMS028223 (diente), 387-208 cal AC, D-AMS028224 (diente); (C) pelo humano ID016, 361-147 cal AC, D-AMS 028226 (pelo); (D) miniatura cerámica con contenido de pop-corn (flecha), 428-573 cal DC, D-AMS 028227 (pop-corn); (E) cesto miniatura con pelo animal (flecha) y miniatura de manta (flecha); 428-573 cal DC, D-AMS 028227 (pelo animal); 479-630 cal DC, D-AMS 028220 (textil); (F) manta fibra animal y algodón, reps de urdimbre, textil ID030, Registro $\mathrm{N}^{\circ} 28$ Canchones UNAP; (G) escápula no perteneciente a ID016, Inventario No B2138 FACSO, 376-522 cal DC, D-AMS 028225 (hueso); (H) cordelería, Inventario No B2138 FACSO, 475-630 cal DC, D-AMS 028218 (cordelería); (I) tobilleras piel de camélido, textil ID440, Inventario $\mathrm{N}^{\circ}$ A05916 FACSO; (J) detalle bolsa textil, anillado cruzado, ID221, Registro $\mathrm{N}^{\circ} 1059$ Canchones UNAP. Fechas calibradas mediante OxCal 4.3, curva SHCal 13 y expresadas con 2 sigmas.

Sample of materials from Tomb 1 Section L, Tr-40 Cemetery. (A and B) Frontal and lateral view of the skull ID016, male-17 years old, Inventory $N^{\circ}$ B2138 FACSO, 370-205 cal BP, D-AMS028223 (tooth), 387-208 cal BP, D-AMSO28224 (tooth); (C) human hair ID016, 361-147 cal BP, D-AMS 028226 (hair); (D) ceramic miniature with pop-corn content (arrow), 428-573 cal AD, D-AMS 028227 (pop-corn); (E) miniature basket with animal hair (arrow) and miniature blanket (arrow); 428-573 cal AD, D-AMS 028227 (animal hair); 479-630 cal AD, D-AMS 028220 (textile); $(F)$ animal fiber and cotton blanket, warp reps, textile ID030, Record $N^{o} 28$ Canchones UNAP; $(G)$ scapula not belonging to ID016, Inventory N $N^{o}$ B2138 FACSO, 376-522 cal AD, D-AMS 028225 (bone); (H) cordage, Inventory $N^{\circ}$ B2138 FACSO, $475-630$ cal AD, D-AMS 028218 (cordage); (I) camelid leather anklets, textile ID440, Inventory $N^{\circ}$ A05916 FACSO; (J) textile bag detail, cross-ringed, ID221, Registration $N^{\circ} 1059$ Canchones UNAP. Dates calibrated using OxCal 4.3, SHCal 13 curve and expressed with 2 sigmas. 
Tabla 3. Frecuencia de tumbas-cuerpos del cementerio Tarapacá 40 referidas en la literatura disponible. Tombs-bodies frequencies from $\mathrm{Tr}$-40 cemetery referred to in available literature.

\begin{tabular}{|c|c|c|c|c|c|}
\hline $\begin{array}{l}\text { Año campaña de } \\
\text { excavación }\end{array}$ & Sector & Sección & $\mathrm{N}^{\circ}$ tumbas-cuerpos & Edad & Referencias \\
\hline $\begin{array}{l}1967-68 \\
1980-81 \\
\end{array}$ & Tr-40A & M'/"bordes" & $100 / 8$ & $\begin{array}{l}58 \text { adultos } \\
36 \text { párvulos y neonatos }\end{array}$ & \multirow{3}{*}{$\begin{array}{l}\text { Núñez 1969; 1970, 1981, 1982; } \\
\text { Núñez et al. 2006; Oakland } 2000\end{array}$} \\
\hline $1967-68$ & $\operatorname{Tr}-40 \mathrm{~B}$ & No-M' & 16 & $?$ & \\
\hline Total & & & 124 & & \\
\hline
\end{tabular}

también por una sobrerrepresentación de individuos, debido a que en este estudio un número de entre seis y ocho partes de cuerpos sin referencia fueron leídos como conjuntos independientes e incompletos.

Antes de esta investigación, la composición sexual y etaria de Tr-40 describía una relación de $58 \%(\mathrm{n}=26)$ para individuos femeninos y $36 \%(\mathrm{n}=16)$ masculinos, en tanto que solo el $12 \%(\mathrm{n}=6)$ estaba adscrito al grupo no-adulto (Arias 2010; Arias y Herrera 2012). Con este trabajo esas cifras se han modificado, mostrando una relación que prácticamente reparte en tres tercios a individuos femeninos, masculinos y no-adultos $(<15$ años), muy similar a la composición de cementerios como Cáñamo 3 en la costa de Tarapacá durante el periodo Formativo (Arias y Herrera 2012; Núñez y Moragas 1977). A pesar de la baja disponibilidad de coxales de individuos adultos para su observación directa (24\%), consideramos que la determinación de sexo logró resultados eficaces gracias a la aplicación de un conjunto amplio de métodos que buscó, precisamente, sortear esta clase de dificultades en la integridad de la muestra.

$\mathrm{Si}$ entonces consideramos a la serie esqueletal de Tr-40 como representativa de la totalidad del cementerio, la presencia de todos los grupos etarios y la proporción cercana a 1:1 entre individuos masculinos y femeninos sugiere que no habría un acceso diferencial por edad o sexo a este espacio funerario; lo que no descarta exclusiones en función de otras categorías.

\section{Tarapacá 40 en el escenario regional: Comunidad, Parentesco y Reproducción}

En una primera lectura, los sitios del interior o relacionados con ellos como Tiliviche-2, Tr-40 y Cáñamo 3, mostrarían una menor mortalidad femenina adulta respecto a los cementerios de la costa (Figura 5), así como una mayor fertilidad, representada en la alta mortalidad no-adulta. Los elementos que influirían en esta variación podrían ser, entre otros, mejoras en los conocimientos técnicos del trabajo de parto y los cuidados de la gestación y el puerperio, cuestión que ha sido planteada con anterioridad sobre la base de evidencia alternativa (Arriaza et al. 1984, Arriaza et al. 1988). Hablamos de "mejoras", en el entendido de que las complicaciones del embarazo, parto y puerperio se hallan entre las principales causas de la mortalidad femenina en edad reproductiva en sociedades pre y no industriales (Fortney et al. 1987; Stone 2016).

De esta manera, una variación dicotómica en la mortalidad como la que se observa entre los cementerios comparados, podría deberse a cambios en el control de aquel factor de mortalidad. No obstante, si bien un mayor conocimiento, asistencia y/o cuidado durante los trabajos de gestación, parto y puerperio, puede tener un impacto en la sobrevida femenina, no necesariamente impacta en la mortalidad infantil y juvenil, la cual está caracterizada por la alta incidencia de enfermedades infecciosas respiratorias y gastrointestinales en esta etapa (Allison et al. 1981; Allison et al. 1979; De Araújo et al. 1985; Fontana et al. 1983; Holden y Núñez 1993; Rothhammer et al. 1985).

Cabe destacar que uno de los estímulos más importantes para buscar mejoras en los saberes asociados al parto y el puerperio es el incremento en la preocupación social por la reproducción, especialmente, cuando las alianzas dependen de ella. Una mujer que sobrevive a un parto, es una mujer que puede embarazarse nuevamente, mientras que un infante o niña/o muerto, puede ser reemplazado por siguientes embarazos. El esfuerzo se orienta a resguardar el umbral de vulnerabilidad del cuerpo que repone la pérdida: el cuerpo femenino. Esto es particularmente importante para aquellos grupos organizados en linajes segmentarios o comunidades domésticas (Meillassoux 1982; Sahlins 2014 [1961]; Tabet 2018), cuya autarquía ocurre a nivel productivo, pero no reproductivo (Carrasco et al. 2011). 
Como ha sido planteado anteriormente (Adán et al. 2007, Uribe 2008), los asentamientos de la porción baja de la quebrada de Tarapacá, asociados a Tr-40, como Pircas o Caserones, muestran ser unidades autónomas que reproducen ideales identitarios mediante el recurso a la ancestría como un modo de reafirmación territorial y política.

Una de las soluciones propuestas para entender estas formaciones sociales, ha sido la consideración de la movilidad no solo altitudinal de las unidades reproductivas, sino también longitudinal (Pimentel et al. 2011). Efectivamente, esta movilidad pudo haber estado estimulada por intereses económicos y políticos, entre los cuales, deben incluirse no solo bienes y productos, sino también aquellos orientados a garantizar el trabajo procreativo y de cuidados, habitualmente, realizados mediante el intercambio de mujeres jóvenes entre comunidades que sostienen deudas o alianzas. Existen algunas evidencias complementarias para sustentar esta hipótesis, como las huellas isotópicas de un cuerpo femenino que habría nacido en la costa y muerto en Tarapacá (Santana et al. 2012).

Por su parte, propuestas recientes sostienen que los rituales festivos y funerarios ocuparon un "lugar central" en los asentamientos con arquitectura pública como Caserones y Guatacondo, cuyos sistemas de organización arquitectónica reforzarían la idea de sistemas sociales segmentarios (Adán et al. 2007; Albarracín-Jordán 1996). Estos planteamientos son coherentes con la producción de un espacio funerario que incluye a gran parte de la comunidad en un aparente interés por representar un ideal de integración/igualdad. Desde este punto de vista, deberíamos concebir a Tr-40 como una necrópolis o lugar de los muertos de la comunidad, a quienes esta les debe culto y, por lo tanto, como un espacio socio-político a través del cual la ideología de la ancestría es actualizada, normalizada y reproducida.

Cuando los colectivos no-adultos son incorporados en esta representación, particularmente los infantes (menores de 12 meses de edad) (Scott 1999), es posible sospechar que se ha desarrollado una preocupación social por las reglas de filiación, es decir, por las relaciones de dependencia y propiedad entre las generaciones (Lull et al. 2005; Meillassoux 1982). Cuando aquello se instala, las relaciones de parentesco devienen en el principal mecanismo de la reproducción social, ya que regulan el producto de los acoplamientos (hijos e hijas) esperado de las alianzas (matrimonios), el cual es reclamado por el segmento o linaje, normado por la política y naturalizado por la cultura. Es lo que Tabet llama la "reproducción forzada", pues es una sanción social la que define las formas, ritmos y destino de las relaciones sociales de reproducción (Tabet 1996, 2018). De esa regulación dependerá el destino del trabajo acumulado y la organización comunitaria entre los segmentos en el ciclo siguiente o la apropiación del producto del trabajo de ciclos previos, entre los cuales debemos incluir a los trabajos procreativos.

En Tr-40 existen numerosas evidencias que sustentan la idea de una comunidad fuertemente enraizada en un culto hacia la ancestralidad, principal evidencia de una organización social sustentada en el parentesco. Esta organización parece desdibujarse hacia un proceso de centralización política hacia finales del periodo, con cambios que son coherentes con un incremento de la dependencia agrícola y la violencia física (Herrera et al. 2017; Vidal Elgueta et al. 2019).

La ritualización y transformación de múltiples elementos de origen arcaico en "patrimonio ancestral" (Agüero y Uribe 2015), como los turbantes y sus objetos asociados, las técnicas textiles en torzal, el uso de faldellines y la cestería en espiral, entre otros, constituyen testimonios elocuentes de una voluntad por reforzar la reproducción de una comunidad que se identifica en función de la representación de un antepasado y un pasado (Uribe et al. 2015). En esta representación, el antepasado figura ideológicamente como referente a quien se le adeuda la gracia de la producción y el saber, de manera que su veneración debe asegurarse para la buenaventura futura. En este sitio, por ejemplo, datamos una tumba con fechas distantes en varios siglos, en la cual se ofrecieron objetos y comidas en torno a 800 años después de la muerte de un individuo, quizás considerado el fundador de un clan o linaje (Figura 6). No obstante, económica y políticamente, el culto al antepasado refleja su reconocimiento como sostenedor de los fundamentos de las alianzas y de la identidad y, por lo tanto, quien permitió establecer la diferencia y la definición de los contornos de la alteridad respecto a otras comunidades. El culto a la ancestría, entonces, conforma el núcleo del orden simbólico que sostiene al sistema de reproducción social regulado por el parentesco.

\section{El grupo no-adulto en el espacio funerario}

La incorporación del colectivo no-adulto al espacio funerario, especialmente aquel menor de un año, es la 
otra cara del culto a la ancestralidad, particularmente, en comunidades domésticas compuestas por segmentos reproductivos como la que pueden haber funcionado en Pircas y Caserones (Meillassoux 1982). La infancia es la que adeudará y garantizará que se cumplan las alianzas establecidas entre sus segmentos. Esa existencia, entonces, deja de ser un asunto doméstico y pasa a ser una cuestión de preocupación pública que debe resolverse a nivel trans-comunitario (Carrasco et al. 2019). Su requerimiento y su pérdida es de un orden supra reproductivo, ya que se encuentra en el fundamento de los compromisos establecidos en las alianzas (Benería 1981). Aquí es donde podemos complementar la primera hipótesis respecto al interés en el desarrollo de mejoras técnicas vinculadas con los trabajos procreativos, ya que un incremento en la fecundidad necesariamente supone un aumento en el trabajo de los cuerpos femeninos. Y, un aumento del trabajo no compensado en el producto y el consumo social, históricamente ha devenido en restricciones de acceso a los espacios públicos de la política (Kurnitzky 1992; Tabet 2005).

Ciertamente, la baja presencia de estos colectivos en los cementerios de la costa parece ilustrativa de comunidades cuyo sistema de cohesión social se establece sobre la base de relaciones de adhesión y no de filiación; donde la muerte de un infante o una persona no-adulta es una cuestión quizás personal o del grupo más próximo, antes que un hecho sancionado por la comunidad. Básicamente, no ha irrumpido un "pater" que lo reclame ni un "gran pater" que lo regule (Strassmann y Garrard 2011). En esa línea, por ejemplo, se ha entendido el trabajo invertido en la momificación de infantes Chinchorro; probablemente, una práctica íntima de saberes reproducidos en la genealogía de la madre y su círculo cercano (Standen et al. 2014).

En este escenario, Tiliviche-2, aparece como un caso bisagra entre estos cementerios, al tratarse de grupos costeros que experimentan con estadías cada vez más prolongadas en el interior. Lugares ya conocidos, pero que se transforman en espacios de uso más permanente y cuya territorialidad, por lo tanto, se vuelve un asunto que debe enfrentarse políticamente. El gran porcentaje de personas noadultas en el cementerio, muy posiblemente, es la manifestación de un aumento de la mortalidad; quizás, debido a la vulnerabilidad de respuesta de este colectivo frente a crisis alimentarias, conflictos bélicos u otros problemas. Pero, también es la manifestación de un aumento de la fecundidad. Desde esta perspectiva,
Tr-40 y Cáñamo 3 podrían ser la manifestación de grupos que han resuelto pacíficamente los asuntos de reproducción a través de la instauración normativa del parentesco. Para que ello ocurra, deben implementarse mecanismos materiales y simbólicos que permitan enfrentar la resistencia a la dominación y la reproducción del nuevo sistema. Habitualmente, se trata de procesos de larga duración que se van instalando a través de normativas acumulativas que a la larga logran consolidar una nueva cultura de la reproducción, es decir, nuevos órdenes simbólicos que naturalizan la división sexual del trabajo y los espacios de la política (Tabet 2018).

\section{Conclusiones}

Con los resultados de este trabajo se ha alcanzado el objetivo de fortalecer la información de origen para establecer la composición sexual y etaria de la serie bioantropológica de la colección de Tr-40. La determinación de sexo y la estimación de edad para la totalidad de la serie bioantropológica permitirán realizar una reconstrucción paleodemográfica y profundizar el estudio de la gestión de la reproducción entre las sociedades formativas tarapaqueñas en los Andes Centro Sur.

No obstante, es importante destacar algunos elementos que obligan una lectura cautelosa de estos resultados. Primero, recordar que la composición sexual y etaria de una serie funeraria no es sinónimo de reconstrucción paleodemográfica (Séguy y Buchet 2013:Figura 2.1). Segundo, que los cuerpos infantiles se encuentran fuertemente afectados por las prácticas funerarias y por factores tafonómicos, los cuales pueden construir falsos negativos (Saunders 2008). Tercero, que como hemos constatado en Tr-40, los procesos de excavación y el manejo de colecciones pueden verse afectados por sesgos muestrales derivados de los intereses y prioridades científicas. Cuarto, que los criterios analíticos son desiguales o no explícitos en las publicaciones consultadas, muchas de las cuales son ambiguas respecto a los universos de estudio y sus muestras. Y, por último, el ajuste cronológico. Por una parte, las fechas suelen ser escasas, generalmente de muestras indirectas y de vida larga (p.ej., carbones y/o maderas), que exhiben lapsos de tiempo extremadamente amplios (Castro Martínez et al. 1993-94). Por otra, dificultades propias del efecto reservorio observado en algunas muestras de las costas del Norte Árido (Carré et al. 2017; Latorre et al. 2017; Ortlieb et al. 2017; Santana-Sagredo et al. 
2017). En consecuencia, la propuesta explicativa que hemos ofrecido en este trabajo debe ser entendida como preliminar y con valor de hipótesis, y como tal busca abrir el debate y estimular preguntas de investigación en torno a materias habitualmente marginalizadas en las ciencias sociales en general.

Por su parte, el acercamiento y el uso de múltiples indicadores de sexo-edad han permitido realizar una identificación mejorada de estas características en la serie de 149 cuerpos de la colección Tr-40. Existe una representación de toda la variedad sexual y etaria en los componentes de la colección, lo que ofrece fuentes de información osteobiográfica y paleodemográfica interesantes. El grupo de adultos constituye cerca del 50\% del total de la muestra, distribuidos en una relación 1:1.

Aunque los resultados del registro de indicadores de sexo y edad, su determinación y estimación pueden ser valorados como positivos, es necesario destacar que los indicadores empleados se caracterizan por una aproximación tipológica y lineal a los procesos degenerativos ocurridos en el esqueleto y su relación cronológica (Bocquet-Appel y Masset 1982; Buckberry 2015; Cardoso et al. 2014a). Es deseable, en consecuencia, la complementación del registro de indicadores de edad mediante métodos transicionales y la comparación de los resultados entre estas distintas aproximaciones (Luna 2018).

No obstante, esta valoración bioarqueológica de la composición sexual y etaria de la colección, nos permite situar a $\mathrm{Tr}-40$ dentro de un grupo de cementerios formativos y del interior de la Pampa del Tamarugal que presentan cierto equilibrio en la proporción de estos parámetros, destacando la inclusión de las personas noadultas en el espacio funerario. Al contrario, cementerios costeros y de fechas más tempranas, parecen excluirlos y/o manifestar menos nacimientos.

A partir de estos resultados hemos sugerido las primeras hipótesis respecto a una forma alternativa de comprender la trayectoria histórica de las comunidades de la Pampa del Tamarugal, considerando trabajos, agentes y relaciones sociales hasta ahora desconocidos en nuestra representación del pasado prehispánico del Norte Árido.

Sugerimos que en la costa arcaica primarían relaciones de adhesión, características de grupos con alta movilidad y de tamaño pequeño, muy probablemente comunidades matrilineales y/o locales. Entre ellos, las relaciones sociales de reproducción se resolverían a nivel del grupo o personalmente, sin establecerse normas ni propiedad sobre el producto de los acoplamientos, y probablemente sin requerir el establecimiento de matrimonios que garantizaran una exposición permanente al riesgo de embarazos. Por su parte, cementerios como Tr-40 mostrarían a una sociedad que se articula sobre principios de parentesco, es decir, regulando el destino de la descendencia a través de la ancestría y el control sobre la sexualidad de las mujeres mediante el establecimiento de matrimonios intercomunitarios, en un modelo muy probablemente patrilocal y/o patrilineal, que aseguraría el resguardo de las alianzas para la vigilancia de la propiedad territorial comunitaria. Proponemos que, de ser así, esta transformación podría sentar las bases de las relaciones estructurales de sexo en la trayectoria histórica de la Pampa del Tamarugal, instalando los órdenes simbólicos que sostendrían la conformación de una clase subalterna identificada con los trabajos procreativos y el imaginario de la maternidad, cuya incidencia política ha de buscarse en coordenadas alternativas a la institucionalidad pública.

Agradecimientos: Agradecemos a quienes participaron como revisores anónimos del manuscrito de este trabajo por sus importantes comentarios y observaciones, varios de los cuales nos permitieron explayarnos sobre materias centrales de nuestra propuesta, no obstante, cualquier error u omisión es de nuestra completa responsabilidad. A Nicole Barreaux, conservadora de FACSO, a Lorena Sanhueza, responsable del Área de Patrimonio de FACSO y Miriam Pérez, coordinadora de Antropología Física de la misma Institución, por facilitarnos el acceso a la colección y ofrecernos todos los espacios de trabajo necesarios. A Álvaro Carevic de la UNAP, por el acceso a la colección Canchones y por brindar toda la infraestructura requerida para los trabajos de laboratorio y estancia de analistas. Al profesor Eugenio Aspillaga por la información histórica de la colección bioantropológica de $\mathrm{Tr}-40$. Gratitud y reconocimiento a todas las personas que trabajaron en el registro bioantropológico, radiológico y contextual de la colección bioantropológica de Tr-40: Iván Arregui, María Cecilia Espinoza, Rocío González, Karmina Jara, Aryel Pacheco, Constanza Pino, Constanza Urrutia, Ignacia González, Francisca Jara, Daniela Rivera y Paula Menanteau. Este trabajo fue financiado por el proyecto FONDECYT Postdoctorado 3160759 y cuenta con apoyo del FONDECYT Regular 1181829. 


\section{Referencias Citadas}

Adán, L., S. Urbina y M. Uribe 2007. Arquitectura pública y doméstica en las quebradas de Pica-Tarapacá: Asentamiento y dinámica social en el Norte Grande de Chile (900-1450 DC). En Procesos Sociales Prehispánicos en el Sur Andino. La Vivienda, la Comunidad y el Territorio, editado por A. Nielsen, C. Rivolta, V. Seldes, M.M. Vázquez y P. Mercolli, pp. 183-206. Editorial Brujas, Córdoba.

Agüero, C. 2012. Textiles del Asentamiento Caserones y su Cementerio: Significado Social y Político para la Población Tarapaqueña durante el Período Formativo (Norte de Chile). Revista Chilena de Antropología 26:59-94.

Agüero, C., M. Uribe. 2015. Tombs and Tumuli on the Coast and Pampa of Tarapacá Explaining the Formative Period in Northern Chile (South-Central Andes). En Funerary Practices and Models in the Ancient Andes: The Return of the Living Dead, editado por P. Eeckhout y L. Owens, pp. 55-68. Cambdridge University Press, New York.

Albarracín-Jordán, J. 1996. Tiwanaku, Arqueología Regional y Dinámica Segmentaria. Plural Editores, La Paz.

Allison, M.J., E. Gerszten, J. Munizaga, C. Santoro y D. Mendoza 1981. Tuberculosis in pre-Columbian Andean populations. En Prehistoric Tuberculosis in the Americas, editado por J. Buikstra, pp. 49-61. Northwestern University Archeological Program, Evanston.

Allison, M.J., E. Gerszten, J. Shadomy, J. Munizaga y M. Gonzalez 1979. Paracoccidioidomycosis in a Northern Chilean mummy. Bulletin of the New York Academy of Medicine 55 (7):670-683.

Anderson, A. y R.F. Loeser 2010. Why is osteoarthritis an agerelated disease? Best Practice \& Research: Clinical Rheumatology 24 (1):15-26.

Arias, M. 2010. Colecciones bioantropológicas y análisis de estado de salud y dieta en una población formativa de la costa desértica de interfluvio Iquique-Loa. Informe de Práctica Profesional, Departamento de Antropología, Universidad de Chile, Santiago.

Arias, M. y M.J. Herrera 2012. Caracterización del modo de vida, salud y dieta de las poblaciones de los cementerios CaseronesTarapacá 40 y Cáñamo 3 (Período Formativo, Región de Tarapacá). Actas del XVIII Congreso Nacional de Arqueología Chilena, pp. 53-62. Sociedad Chilena de Arqueología, Valparaíso.

Arnold, D. 1997. Más allá del Silencio. Las Fronteras de Género en los Andes. ILCA/CIASE, La Paz.

Arriaza, B., M. Allison, G. Focacci y E. Gerszten 1984. Mortalidad materna y de la niñez en el área de Arica Prehispánica y conceptos asociados. Chungara Revista de Antropología Chilena 12:161-172.

Arriaza, B., M. Allison y E. Gerszten 1988. Maternal mortality in pre-Columbian Indians of Arica, Chile. American Journal of Physical Anthropology 77 (1):35-41.

Bajwa, M., D. Srinivasan, H. Nishikawa, D. Rodrigues, G. Solanki y N. White 2013. Normal fusion of the metopic suture. The Journal of Craniofacial Surgery 24 (4):1201-1205.

Becker, M.J. 1986. Mandibular symphysis (medial suture) closure in modern Homo sapiens: preliminary evidence from archaeological populations. American Journal of Physical Anthropology 69 (4):499-501.
Benería, L. 1981. Reproducción, producción y división sexual del trabajo. Mientras Tanto 6:47-84.

Bocquet-Appel, J.-P. y C. Masset 1982. Farewell to Paleodemography. Journal of Human Evolution 11:321-333.

Bogin, B. y B.H. Smith 1996. Evolution of the human life cycle. American Journal of Human Biology 8:703-716.

Bourdieu, P. 2011. Las Estrategias de la Reproducción Social. Siglo XXI, Buenos Aires.

Boydston, J. 1990. Home and Work: Housework, Wages, and the Ideology of Labor in the Early Republic. Oxford University Press, New York.

Brooks, S. y J.M. Suchey 1990. Skeletal age determination based on the os pubis: a comparison of the Acsadi-Nemeskeri and Suchey-Brooks methods. Human Evolution 5 (3):227-238.

Brothwell, D.R. 1981. Digging up Bones: the Excavation, Treatment, and Study of Human Skeletal Remains. Cornell University Press, Ithaca.

Buckberry, J. 2015. The (mis)use of adult age estimates in osteology. Annals of Human Biology 42 (4):323-331.

Cardoso, H.F. 2008a. Age estimation of adolescent and young adult male and female skeletons II, epiphyseal union at the upper limb and scapular girdle in a modern Portuguese skeletal sample. American Journal of Physical Anthropology 137 (1):97-105.

Cardoso, H.F. 2008b. Epiphyseal union at the innominate and lower limb in a modern Portuguese skeletal sample, and age estimation in adolescent and young adult male and female skeletons. American Journal of Physical Anthropology 135 (2):161-170.

Cardoso, H.F., J. Abrantes y L.T. Humphrey 2014a. Age estimation of immature human skeletal remains from the diaphyseal length of the long bones in the postnatal period. International Journal of Legal Medicine 128 (5):809-824.

Cardoso, H.F., J. Gomes, V. Campanacho y L. Marinho 2013. Age estimation of immature human skeletal remains using the post-natal development of the occipital bone. International Journal of Legal Medicine 127 (5):997-1004.

Cardoso, H.F., V. Pereira y L. Ríos 2014b. Chronology of fusion of the primary and secondary ossification centers in the human sacrum and age estimation in child and adolescent skeletons. American Journal of Physical Anthropology 153 (2):214-225.

Cardoso, H.F. y L. Ríos Frutos 2011. Age estimation from stages of epiphyseal union in the presacral vertebrae. American Journal of Physical Anthropology 144 (2):238-247.

Carrasco, A.M. y V. Gavilán 2009. Representaciones del cuerpo, sexo y género entre los aymara del norte de Chile. Chungara Revista de Antropología Chilena 41 (1):83-100.

Carrasco, C. 1991. El Trabajo Doméstico y la Reproducción Social. Instituto de la Mujer, Madrid.

Carrasco, C., C. Borderías y T. Torns (eds.) 2019. El Trabajo de Cuidados: Historia, Teoría y Políticas. Catarata, Madrid.

Carrasquer, P. 2013. El redescubrimiento del trabajo de cuidados: algunas reflexiones desde la sociología. Cuadernos de Relaciones Laborales 31 (1):91-113. 
Carré, M., D. Jackson, A. Maldonado, B.M. Chase y J.P. Sachs 2017. Variability of $14 \mathrm{C}$ reservoir age and air-sea flux of CO2 in the Peru-Chile upwelling region during the past 12,000 years. Quaternary Research 85 (1):87-93.

Castro Martínez, P.V., R.W. Chapman, S. Gili Suriñach, V. Lull, R. Micó Pérez, C. Rihuete Herrada, R. Risch y M.E. Sanahuja Yll 1993-94. Tiempos sociales de los contextos funerarios argáricos. AnMurcia 9-10:77-105.

Castro-Martínez, P.V., C. Rihuete Herrada y R. Micó Pérez 1995. La Prehistoria Reciente en el Sudeste de la Península Ibérica: Dimensión socio-económica de las prácticas funerarias. En Arqueoloxía da morte: Arqueoloxía da Morte na Península Ibérica desde as Orixes ata o Medievo, coordinado por C. Fernández, F. Pérez y R. Fábregas, pp. 127-168. Concello de Xinzo de Limia, Xinzo de Limia.

Castro, P.V., R.W. Chapman, S. Gili Surinach, V. Lull, R. Micó Pérez, C. Rihuete Herreda, R. Risch y M.E. Sanahuja Yll 1996. Teoría de las prácticas sociales. Complutum Extra 6 (II):35-48.

Cocilovo, J.A., S. Quevedo y H. Varela 1999. Biología del grupo prehistórico de Pisagua, costa norte de Chile. Estudios Atacameños 17:207-235.

Cocilovo, J.A., H. Varela, M.A. Costa-Junqueira y S. Quevedo 2005. Los pescadores arcaicos de la desembocadura del río Loa (norte de Chile): El sitio caleta Huelén 42. Chungara Revista de Antropología Chilena 37 (1):5-19.

Costa, M.A., H.H. Varela, J.A. Cocilovo, S. Quevedo y S.G. Valdano 2000. Perfil paleodemográfico de Morro de Arica, una población de pescadores arcaicos del norte de Chile. Boletín del Museo Nacional de Historia Natural (Chile) 49:215-235.

Dalla Costa, M. 1975. Las mujeres y la subversión de la comunidad. En El Poder de la Mujer y la Subversión de la Comunidad, editado por S. James y M. Dalla Costa, pp. 22-65. S. XXI, México DF.

De Araújo, A., L.F. Ferreira, U. Confalonieri, L. Nuñez y B.M. Ribeiro Filho 1985. The finding of Enterobius vermicularis eggs in pre-Columbian human coprolites. Memorias do Instituto Oswaldo Cruz 80 (2):141-143.

Dean, C. 2001. Andean androgyny and the making of men. En Gender in Pre-Hispanic America, editado por C. Klein, J. Quilter, pp. 143-182. Dumbarton Oaks Researsh Library, Washington, D.C.

Delphy, Ch. 1987. Modo de producción doméstico y feminismo materialista. En Mujeres: Ciencia y Práctica Política, compilado por C. Amorós, pp. 17-28. Debate, Madrid.

Escoriza Mateu, T. 2006. Mujeres, vida social y violencia. Política e ideología en el arte rupestre levantino. Cypsela 16:19-36.

Falquet, J. 2018. Introducción Paola Tabet: desnaturalizando radicalmente la situación de las mujeres. En Los Dedos Cortados, de P. Tabot, traducción A. Cuenca, compilación y edición académica J. Falquet, pp. 27-49. Editorial Universidad Nacional de Colombia, Bogotá.

Federici, S. 2010. Calibán y la Bruja. Mujeres, Cuerpo y Acumulación Originaria. Traficantes de sueños, Madrid.

Federici, S. 2018. El Patriarcado del Salario. Críticas Feministas al Marxismo. Traficantes de sueños, Madrid.
Ferembach, D., I. Schwidetzky y M. Stloukal 1980. Recomendations for age and sex diagnoses of skeletons. Journal of Human Evolution 9:517-549.

Fontana, D., M.J. Allison, E. Gerszten y B. Arriaza 1983. Enfermedades respiratorias agudas en los habitantes precolombinos del Norte Grande chileno. Chungara Revista de Antropología Chilena 11:153-160.

Fortney, J.A., S. Gadalla, S. Saleh, I. Susanti, M. Potts y S.M. Rogers 1987. Causes of death to women of reproductive age in two developing countries. Population Research and Policy Review 6 (2):137-148.

González, I., P. Menanteau y D. Rivera 2017. Bioarqueológico Cáñamo 3, Proyecto FONDECYT de Postdoctorado 3160759 , Universidad de Chile. Informe disponible en FONDECYT, Santiago.

González-Ramírez, A. 2019. Representaciones arqueológicas de la reproducción social: economía y relatos de la gestación y el cuidado en la Pampa del Tamarugal (ca. 2000 cal ANE-900 cal DNE). Proyecto FONDECYT de Postdoctorado 3160759 , Universidad de Chile. Informe disponible en FONDECYT, Santiago.

González-Ramírez, A., A. Pacheco Miranda, A. Sáez e I. Arregui Wunderlich 2019. Infants from the Tarapacá 40 cemetery (Northern Chile, Formative Period, 1000 BC-AD 600). International Journal of Osteoarchaeology 29 (5):874-880.

González-Ramírez, A. y A. Sáez 2011. Aportes para una bioarqueología social y feminista. Revista Atlántica-Mediterránea de Prehistoria y Arqueología Social 13:81-96.

Gowland, R. y C. Knüsel 2006. Social Archaeology of Funerary Remains. Oxbow Books, Oxford.

Herrera, M.J. 2010. Caracterización de los modos de vida y análisis de salud y dieta a través de piezas óseas y dentales en esqueletos del Cementerio Caserones-Tarapacá 40 (periodo Formativo, Región de Tarapacá). Informe de Práctica Profesional, Departamento de Antropologia, Universidad de Chile, Santiago.

Herrera, M.J., R. Retamal, A. Pacheco, P. Díaz, F. Santana y M. Uribe 2017. Muerte violenta de una mujer del Formativo tarapaqueño, norte de Chile (1000 AC-900 DC): una perspectiva bioarqueológica. VII Reunión de la Asociación de Paleopatología en Sudamérica, Vida y Muerte en el Desierto de Atacama, pp. 188-189 Instituto de Alta Investigación, Arica.

Holden, T.G. y L. Núñez 1993. An Analysis of the Gut Contents of Five Well-Preserved Human Bodies from Tarapacá, Northern Chile. Journal of Archaeological Science 20 (6):595-611.

Irurita, J. e I. Aleman 2017. Proposal of new regression formulae for the estimation of age in infant skeletal remains from the metric study of the pars basilaris. International Journal of Legal Medicine 131 (3):781-788.

Iscan, M.Y., S.R. Loth y R.K. Wright 1984. Metamorphosis at the sternal rib end: A new method to estimate age at death in white males. American Journal of Physical Anthropology 65 (2): $147-156$

Kimura, K. 1982. A base-wing index for sexing the sacrum. Journal of the Anthropological Society of Nippon 90 (Supplement):153-162. 
Kuhn, T. 2006. La Estructura de las Revoluciones Científicas. Fondo de Cultura Económica, México, D.F.

Kurnitzky, H. 1992. La Estructura Libidinal del Dinero: Una Contribución a la teoría de la Femineidad. Siglo XXI, México, D.F.

Latorre, C., R. De Pol-Holz, C. Carter y C.M. Santoro 2017. Using archaeological shell middens as a proxy for past local coastal upwelling in northern Chile. Quaternary International 427:128-136.

Lê, S., J. Josse y F. Husson 2008. FactoMineR: An R Package for Multivariate Analysis. Journal of Statistical Software 25 (1):1-18.

Lemp, C., M. Rodríguez, R. Retamal y E. Aspillaga 2008. Arqueología del depósito: manejo integral de las colecciones bioantropológicas en el Departamento de Antropología de la Universidad de Chile. Conserva 12:69-96.

Lovejoy, C.O. 1985. Dental wear in the Libben Population: Its functional pattern and role in the determination of adult skeletal age of death. American Journal of Physical Anthropology 68:47-56.

Lovejoy, C.O., R.S. Meindl, T.R. Pryzbeck y R.P. Mensforth 1985. Chronological metamorphosis of the auricular surface of the ilium: a new method for the determination of adult skeletal age at death. American Journal of Physical Anthropology 68 (1):15-28.

Lull, V., R. Micó Pérez, C. Rihuete Herrada, R. Risch. 2005. Property Relations in the Bronze Age of South-western Europe: an archaeological analysis of infant burials from El Argar (Almeria, Spain). Proceedings of the Prehistoric Society 71:247-268.

Lull, V. y M. Picazo 1989. Arqueología de la muerte y estructura social. Archivo Español de Arqueología 62:5-20.

Luna, L. 2018. Conferencia Clausura. V Reunión Anual Sociedad Chilena de Bioantropología, Museo Nacional de Historia Natural, Santiago.

MacInnes, J. y J. Pérez 2008. La tercera revolución de la modernidad: la revolución reproductiva. Revista Española de Investigaciones Sociológicas (122):89-118.

Mann, R., R. Jantz, W. Bass y P. Willey 1991. Maxillary suture obliteration: a visual method for estimating skeletal age. Journal of Forensic Sciences 36 (3):781.

Mathieu, N.C. 1971. Notes pour une définition sociologique des catégories de sexe. Épistémologie Sociologique 11 (2):19-39.

McGuire, R. 2018. Diálogos con los muertos. Ideología y cementerio. Vestígios-Revista Latino-Americana de Arqueologia Histórica 12 (1):123-158.

Meillassoux, C. 1982. Mujeres, Graneros y Capitales: Economía Doméstica y Capitalismo. Siglo XXI, Mexico, D.F.

Meindl, R.S. y C.O. Lovejoy 1985. Ectocranial suture closure: a revised method for the determination of skeletal age at death based on the lateral-anterior sutures. American Journal of Physical Anthropology 68 (1):57-66.

Méndez-Quiros, P., 2009. "Rescatando la prehistoria tarapaqueña”. Conservación y puesta en valor de las colecciones arqueológicas de La Huayca, Provincia del Tamarugal. Informe Proyecto FONDART N ${ }^{\circ} 72609$.

Miles, A.E.W. 1963. The dentition in the assessment of individual age in skeletal material. En Dental Anthropology, editado por D. Brothwell, pp. 191-209. Pergamon Press, Oxford.
Millet, K. 2010. Política Sexual. Ediciones Cátedra, Madrid.

Molyneux, M. 1979. Beyond the domestic labour debate. New Left Review I (116):3-27.

Munizaga, J., M.J. Allison y E. Aspillaga 1978a. Diaphragmatic hernia associated with strangulation of the small bowel in an Atacamena mummy. American Journal of Physical Anthropology 48 (1):17-19.

Munizaga, J., M.J. Allison y C. Paredes 1978b. Cholelithiasis and cholecystitis in pre-Columbian Chileans. American Journal of Physical Anthropology 48 (2):209-213.

Murra, J. 1987. El control vertical de un máximo de pisos ecológicos en la economía de las sociedades Andinas. En La Teoría de la Complementariedad Vertical Eco-Simbiótica, editado por R. Condarco y J. Murra, pp. 29-85. Hisbol, La Paz.

Narotzky, S. 1995. Mujer, Mujeres, Género: Una Aproximación Crítica al Estudio de las Mujeres en las Ciencias Sociales. CSIC, Madrid.

Núñez, L. 1969. El primer fechado radiocarbónico del complejo Faldas del Morro en el sitio Tarapacá 40 y algunas discusiones básicas. Actas del V Congreso Chileno de Arqueología, pp. 47-57. Dirección General de Bibliotecas Archivos y Museos, La Serena.

Núñez, L. 1970. Algunos problemas del estudio del complejo arqueológico faldas del Morro del norte de Chile. Band Abhandlungen und Berichte des Staatlichen Museums für Völkerkunde 31:79-116.

Núñez, L. 1972. Cambios de Asentamientos Humanos en la Quebrada de Tarapacá: Norte de Chile. Documentos de Trabajo 2, Programa de Arqueología y Museos, Universidad de Chile, Antofagasta.

Núñez, L. 1974. La Agricultura Prehistórica en Los Andes Meridionales. Editorial Orbe, Santiago.

Núñez, L. 1976. Registro regional de fechas radiocarbónicas del norte de Chile. Estudios Atacameños 4:69-111.

Núñez, L. 1981. Emergencia de sedentarización en el desierto chileno. Subsistencia agraria y cambio sociocultural. Creces 2 (11):33-38.

Núñez, L. 1982. Temprana emergencia de sedentarismo en el desierto chileno: proyecto Caserones. Chungara Revista de Antropología Chilena 9:80-122.

Núñez, L. 1984. El asentamiento Pircas: nuevas evidencias de tempranas ocupaciones agrarias en el norte de Chile. Estudios Atacameños. Arqueología y Antropología Surandinas 7:117-134.

Núñez, L. 2006. Asentamientos formativos complejos en el centro-sur andino: cuando la periferia se constituye en núcleo. Boletín de Arqueología PUCP 10:321-356.

Nuñez, L., I. Cartajena, C. Carrasco, P. de Souza y M. Grosjean 2006. Emergencia de comunidades pastoralistas formativas en el sureste de la Puna de Atacama. Estudios Atacameños 32:93-117.

Núñez, L. y T. Dillehay 1979. Movilidad Giratoria. Armonía Social y Desarrollo en los Andes Meridionales: Patrones de Tráfico e Interacción Económica. Universidad Católica del Norte, Antofagasta.

Núñez, L. y C. Moragas 1977. Una ocupación con cerámica temprana en la secuencia del distrito de Cáñamo (costa desértica del norte de Chile). Estudios Atacameños 5:23-50. 
Núñez, L. y C.M. Santoro 2011. El tránsito Arcaico-Formativo en la circumpuna y valles occidentales del centro sur andino: hacia los cambios "neolíticos". Chungara Revista de Antropología Chilena 43 (especial):487-530.

Oakland, A. 2000. Andean textiles from village and cemetery: Caserones in the Tarapaca Valley, North Chile. En Beyond Cloth and Cordage. Archaeological Textile Research in the Americas, editado por P. Ballard y L.D. Webster, pp. 229-251. The University of Utah Press, Salt Lake City.

Oakley, A. 2015. Sex, Gender and Society. Surrey Ashgate Publishing, Ltd., Farnham.

Ortlieb, L., G. Vargas y J.F. Saliège 2017. Marine radiocarbon reservoir effect along the northern Chile-southern Peru coast $\left(14-24^{\circ} \mathrm{S}\right)$ throughout the Holocene. Quaternary Research 75 (1):91-103

Passeron, J.C. y T. García 1983. La teoría de la reproducción social como una teoría del cambio: una evaluación crítica del concepto de contradicción interna. Estudios Sociológicos 1 (3):417-442.

Pearson, M. 1999. The Archaeology of Death and Burial. Sutton, Stroud.

Phenice, T. 1969. A newly developed visual method of sexing the os pubis. American Journal of Physical Anthropology 30 (2):297.

Picchio, A. 2019. La reproducción social y la estructura básica del mercado laboral, en El Trabajo de Cuidados, Historia, Teoría y Políticas, editado por C. Carrasco, C. Borderías y T. Torns, pp. 120-142. Catarata, Madrid.

Pimentel, G., C. Rees, P. de Souza y L. Arancibia 2011. Viajeros costeros y caravaneros. Dos estrategias de movilidad en el Período Formativo del desierto de Atacama, Chile. En En ruta. Arqueología, Historia y Etnografía del Tráfico Sur Andino, editado por L. Nuñez, A. Nielsen, pp. 43-82. Encuentro, Córdoba.

Primeau, C., L. Friis, B. Sejrsen y N. Lynnerup 2016. A method for estimating age of medieval sub-adults from infancy to adulthood based on long bone length. American Journal of Physical Anthropology 159 (1):135-145.

Purkait, R. 2003. Sex determination from femoral head measurements: a new approach. Legal Medicine 5:S347-S350.

Purkait, R. y H. Chandra 2004. A study of sexual variation in Indian femur. Forensic Science International 146 (1):25-33.

R Core Team 2019. R: A language and environment for statistical computing. R Foundation for Statistical Computing, Vienna. Disponible en https://www.R-project.org/.

Retamal, R. y A. Sáez 2006. Efectos de la deformación craneana sobre el dimorfismo sexual en la población chilena prehistórica. Il Quattrocento 1:181-197.

Rihuete, C. 2003. Esqueletos humanos en la investigación arqueológica de la diferencia sexual. En Morir en Femenino: Mujeres, Ideología y Prácticas Funerarias desde la Prehisoria, coordinado por M. Molas i Font y S. Guerra, pp. 18-61, Barcelona.

Ríos Frutos, L. 2002. Determination of Sex from the Clavicle and Scapula in a Guatemalan Contemporary Rural Indigenous Population. The American Journal of Forensic Medicine and Pathology 23 (3):284-288.
Ríos Frutos, L. 2003a. Brief communication: Sex determination accuracy of the minimum supero-inferior femoral neck diameter in a contemporary rural Guatemalan population. American Journal of Physical Anthropology 122 (2):123-126.

Ríos Frutos, L. 2003b. Determinación métrica del sexo en esqueletos rurales indígenas guatemaltecos. XVI Simposio de Investigaciones Arqueológicas en Guatemala, pp. 416-426. Museo Nacional de Arqueología y Etnología, Guatemala.

Ríos Frutos, L. 2005. Metric determination of sex from the humerus in a Guatemalan forensic sample. Forensic Science International 147 (2-3):153-157.

Risch, R. 2002. Análisis funcional y producción social: relación entre método arqueológico y teoría económica. En Análisis Funcional: Su aplicación al Estudio de Sociedades Prehistóricas, editado por I. Clemente, R. Risch y J.F. Gibaja, pp. 19-29. BAR International Series 1073.

Rissech, C. y S. Black 2007. Scapular development from the neonatal period to skeletal maturity: A preliminary study. International Journal of Osteoarchaeology 17 (5):451-464.

Rissech, C., M. García y A. Malgosa 2003. Sex and age diagnosis by ischium morphometric analysis. Forensic Science International 135 (3):188-196.

Rissech, C., O. Lopez-Costas y D. Turbon 2013a. Humeral development from neonatal period to skeletal maturity-application in age and sex assessment. International Journal of Legal Medicine 127 (1):201-212.

Rissech, C. y A. Malgosa 2007. Pubis growth study: applicability in sexual and age diagnostic. Forensic Science International 173 (2-3):137-145.

Rissech, C., N. Marquez-Grant y D. Turbon 2013b. A collation of recently published Western European formulae for age estimation of subadult skeletal remains: recommendations for forensic anthropology and osteoarchaeology. Journal of Forensic Sciences 58 Suppl 1:S163-S168.

Ross, A.H. y M.J. Manneschi 2011. New identification criteria for the Chilean population: Estimation of sex and stature. Forensic Science International 204 (1):206. e201-206. e203.

Rothhammer, F., M.J. Allison, L. Núñez, V. Standen y B. Arriaza 1985. Chagas' disease in pre-Columbian South America. American Journal of Physical Anthropology 68 (4):495-498.

Rothhammer, F., V. Standen, L. Núñez, M.J. Allison y B. Arriaza 1984. Origen y desarrollo de la tripanosomiasis en el área Centro-Sur Andina. Chungara Revista de Antropología Chilena 12: 155-160.

Sáez, A. y A. González-Ramírez 2018. Preservación, momificación y composición sexual y etaria de la serie esqueletal de la Colección Tarapacá 40, Proyecto FONDECYT de Postdoctorado 3160759. Universidad de Chile. Informe disponible en FONDECYT, Santiago.

Sáez, A. y G. Manríquez 2006. Efectos del dimorfismo sexual en la variación morfológica de cráneos de la población chilena. Análisis de morfometría lineal y morfometría geométrica. Actas V. Congreso Chileno de Antropología: Antropología en Chile: Balance y perspectivas, pp. 965-973. Colegio de Antropólogos de Chile, San Felipe. 
Sahlins, M.D. 2014 [1961]. El linaje segmentario: una organización de expansión depredadora. Alteridades 7:99-113.

Sanahuja, E. 2002. Cuerpos Sexuados, Objetos y Prehistoria. Cátedra, Madrid.

Sanahuja, E. 2007. La Cotidianeidad en la Prehistoria: La Vida y su Sostenimiento. Icaria, Barcelona.

Santana-Sagredo, F., R. Schulting, J. Lee-Thorp, C. Agüero, M. Uribe y C. Lemp 2017. Paired Radiocarbon Dating on Human Samples and Camelid Fibers and Textiles from Northern Chile: The Case of Pica 8 (Tarapacá). Radiocarbon 59 (4):1195-1213.

Santana-Sagredo, F., M. Uribe, M.J. Herrera, R. Retamal y S. Flores 2015. Dietary practices in ancient populations from northern Chile during the transition to agriculture (Tarapacá region, 1000 BC-AD 900). American Journal of Physical Anthropology 158 (4):751-758.

Santana, F., M.J. Herrera y M. Uribe 2012. Acercamiento a la paleodieta en la costa y quebradas tarapaqueñas durante el período Formativo: análisis de isótopos estables a partir de tres casos de estudio. Boletín de la Sociedad Chilena de Arqueología 41-42:109-126.

Saunders, S.R. 2008. Juvenile skeletons and growth-related studies. En Biological Anthropology of the Human Skeleton, editado por M.A. Katzemberg y S. Saunders, pp. 117-147. John Willey \& Sons, Inc., Hoboken.

Scheuer, L. y S. Black 2000. Developmental Juvenile Osteology. Academic Press, London.

Scott, E. 1999. The Archaeology of Infancy and Infant Death. BAR International Series 819. Archeopress, Oxford.

Séguy, I. y L. Buchet. 2013. Handbook of Palaeodemography. Springer, London.

Smith, B.H. 1991. Standards of human tooth formation and dental age assessment. En Advances in Dental Anthropology, editado por M. Kelley, C.S. Larsen, pp. 143-168. Wiley-Liss Inc., New York.

Standen, V., B. Arriaza y C. Santoro 2014. Chinchorro mortuary practices on infants: Northern Chile archaic period (BP 7000-3600). En Tracing Childhood: Bioarchaeological Investigations of Early Lives in Antiquity, editado por J. Thompson, M. Alfonso-Durruty, J. Crandall, pp. 58-74. University of Florida Press, Gainesville.

Standen, V. y L. Nuñez 1984. Indicadores antropológico-físico y culturales del cementerio precerámico Tiliviche-2 (Norte de Chile). Chungara Revista de Antropología Chilena 12: 135-153.

Stone, P.K. 2016. Biocultural perspectives on maternal mortality and obstetrical death from the past to the present. American Journal of Physical Anthropology 159 (Suppl 61):S150-S171.

Strassmann, B.I. y W.M. Garrard 2011. Alternatives to the grandmother hypothesis: a meta-analysis of the association between grandparental and grandchild survival in patrilineal populations. Human Nature 22 (1-2):201-222.

Tabet, P. 1985. Fertilité naturelle, reproduction force. En L'Arraisonnment des Femmes. Essais en Antropologie des Sexes, editado por. N.C. Mathieu, pp. 61-146. EHESS, Paris.

Tabet, P. 1996. Natural fertility, forced reproduction. En Sex in Question: French materialist feminism, editado por D. Leonard y L. Adkins, pp. 111-181. Taylor \& Francis, London.

Tabet, P. 2005. Las manos, los instrumentos, las armas. En El Patriarcado Desnudo: Tres Feministas Materialistas, editado por O. Curiel y J. Falquet, pp. 57-129. Brecha Lésbica, Buenos Aires.

Tabet, P. 2018. Los Dedos Cortados. Editorial Universidad Nacional de Colombia, Bogotá.

Todd, T.W. 1920. Age changes in the pubic bone. I. The male white pubis. American Journal of Physical Anthropology 3 (3):285-339.

True, D. 1980. Archaeological investigations in northern Chile: Caserones. En Prehistoric Trails of Atacama: Archaeology of Northern Chile, editado por C.W. Meighan y D.L. True, pp. 139-178. Institute of Archaeology, University of California, Los Angeles.

Tubert, S. (ed.) 2003. Del Sexo al Género: los Equívocos de un Concepto. Cátedra, Madrid.

Uribe, M. 2008. El Formativo: ¿Progreso o tragedia social? Reflexiones sobre evolución y complejidad social desde Tarapacá-(norte de Chile, Andes Centro Sur), en Sed Non Satiata II: Acercamientos sociales en la Arqueología Latinoamericana, editado por F. Acuto, A. Zarankin, pp. 303-324. Editorial Brujas, Córdoba.

Uribe, M., C. Agüero, D. Catalán, M.J. Herrera y F. SantanaSagredo 2015. Nuevos fechados del sitio Tarapacá-40: recientes análisis y reflexiones sobre un cementerio clave del período Formativo del norte de Chile y Andes Centro Sur (1110 a.C.-660 d.C). Ñawpa Pacha 35 (1):57-89.

Vidal Elgueta, A., L.F. Hinojosa, M.F. Pérez, G. Peralta y M.U. Rodriguez 2019. Genetic and phenotypic diversity in 2000 years old maize (Zea mays L.) samples from the Tarapaca region, Atacama Desert, Chile. PLoS ONE 14 (1):e0210369.

Vila-Mitjá, A. 2011. Política y feminismo en arqueología prehistórica. Revista Atlántica-Mediterránea de Prehistoria y Arqueología Social 13:17-32.

Washburn, S. 1948. Sex Differences in the Pubic bone. American Journal of Physical Anthropology 6 (2):199-208.

Weaver, D.S. 1979. Application of the likelihood ratio test to age estimation using the infant and child temporal bone. American Journal of Physical Anthropology 50 (2):263-269.

\section{Notas}

1 Parte del material de $\mathrm{Tr}-40$, fundamentalmente el bioantropológico,

La edad informada se entiende como un estimador del valor central se acopió en estos espacios o contenedores donados por la Fundación Cardoen a la FACSO, Universidad de Chile.

2 Los resultados obtenidos de la recomposición de la información contextual proveniente del material arqueológico y bioantropológico se encuentra en preparación. de probabilidades (no calculadas aquí) a partir del resultado de los distintos métodos de estimación en consideración de su relativa certeza y precisión. Es decir, la edad real del individuo examinado se encuentra en torno a la edad informada. Las fracciones de un año están enunciadas en decimales, de modo que para calcular los meses ha de multiplicarse el dígito decimal 12 veces. 